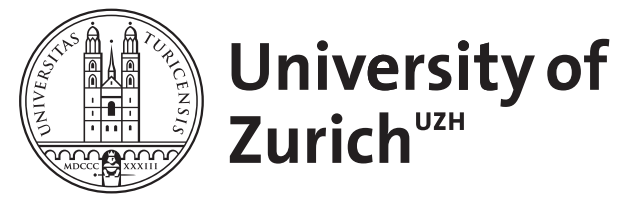

\title{
Städtische Ikonographien, narrative Räume und filmische Geschichtsbilder in
} Gladiator und Rome

\author{
Tröhler, Margrit
}

DOI: https://doi.org/10.1515/9783110400960-014

Posted at the Zurich Open Repository and Archive, University of Zurich ZORA URL: https://doi.org/10.5167/uzh-116384

Book Section

Published Version

Originally published at:

Tröhler, Margrit (2015). Städtische Ikonographien, narrative Räume und filmische Geschichtsbilder in Gladiator und Rome. In: Fuhrer, Therese; Mundt, Felix; Stenger, Jan. Cityscaping : Constructing and Modelling Images of the City. Berlin/Boston: Walter de Gruyter, 281-307.

DOI: https://doi.org/10.1515/9783110400960-014 


\title{
Margrit Tröhler
}

\section{Städtische Ikonographien, narrative Räume und filmische Geschichtsbilder in Gladiator und Rome}

\begin{abstract}
From its inception, cinema has consolidated a vision of ancient Rome based in $18^{\text {th }}$ and $19^{\text {th }}$ century art and literature, condensing it into a topos that informs popular views of antiquity. Period films model their fictional worlds on the basis of iconographic traditions, narrative conventions, and generic patterns, which bear cultural values and perspectives from the outset. Even though the images they project are relatively stable, each new use offers the opportunity not just to invoke the topos, but also to modify it. The films Gladiator and Rome thus add new aspects to the culturally established image of the ancient city. In both cases, the urban space is more than just a backdrop to the events, as it acts upon characters, emotional situations, and plot moments in significant ways. The cinematic mise-en-scène and narrative dynamization of the historic urban space also lead to a partial resemantisation of the locations of power. The essay shows how, in these processes of spatialization, the audiovisual discourse self-reflexively calls attention to itself, revealing two distinct historiographic conceptions.
\end{abstract}

Die Stadt Rom im Historienfilm, genauer im Antikefilm, evoziert von vornherein die Vorstellung von monumentalen Palästen und Plätzen, Tempeln, Paradestraßen, Siegessäulen und prächtigen Interieurs, lichtdurchfluteten Säulenhallen, stillen Atrien sowie Bädern, Gelagen, wallenden Gewändern, üppigen Leibern - und irgendwo im Untergrund: dunklen Verliesen, feuchten Kerkern und angeketteten Gefangenen.

Solche Bilder, ob schwarzweiß oder in Farbe, sind Mosaiksteinchen, die sich zu einem Topos der historischen Stadt Rom verdichten, den das Kino, angelehnt an die Malerei und Literatur des 18. und vor allem 19. Jahrhunderts, seit seinen Anfängen in unseren Köpfen gefestigt und filmisch in Bewegung gesetzt hat, und den es immer wieder überschreibt. Die Antikefilme prägen so das populäre Geschichtsbild einer Stadt und pars pro toto der Antike, ${ }^{1}$ die wir in unserer Erinnerung auch gleich mit großen Männern wie Caesar, Nero oder

1 Zur Entstehung populärer Geschichtsbilder der Antike durch den Schulunterricht vgl. Lagny (1992); hinsichtlich des historischen Romans seit Walter Scott vgl. auch Jameson (1993) 68. 
Spartakus und einigen namhaften Frauen, allen voran Cleopatra, bevölkern. Mehr noch, die spektakuläre und sinnliche Ikonographie der Stadt stellt das Dekor und den narrativen Raum für die Inszenierung historischer Ereignisse und die Taten einzelner Helden. Dieses Stadtbild transportiert den ,Mythos' (im Sinne eines kulturellen Zeichens nach Roland Barthes ${ }^{2}$ ) der Antike als Wiege der westlichen Kultur, in das sich indes auch das melodramatische Muster von rise and fall, d.h. hier von Dekadenz, einschreibt.

Historienfilme modellieren ihre fiktionalen Welten also aufgrund von ikonographischen Traditionen, narrativen Konventionen und Genrevorgaben, die von vornherein kulturelle Wertepositionen beinhalten. Die filmische Gestaltung lädt durch Auswahl, Perspektive und stilistische Parameter die sozialen Lebens- und Handlungsräume urbaner Topographien zusätzlich semantisch auf und unterstreicht symbolische Orte der Macht. Der Umgang mit dem Topos der antiken Stadt Rom lässt dennoch Variationen zu: So kreieren der Kinofilm Gladiator (Ridley Scott, USA 2000) und die amerikanisch-britische Quality Soap Rome (HBO/BBC, 1. Staffel 2005; 2. Staffel 2007) unterschiedliche Stadtbilder. Durch den jeweiligen Einsatz der audiovisuellen Ausdrucksmittel und die spezifische Entwicklung einer zeitlichen Dynamik der Narration entwerfen sie verschiedene diegetische Räume, die die Figuren und ihren Handlungsspielraum im städtischen Umfeld je auf ihre Art prägen. Die Stadt Rom funktioniert dabei als Matrix im Sinne eines abstrakten, imaginären Ganzen, das durch konkrete Räume bestückt wird und dennoch immer lückenhaft bleibt. Die Ausgestaltung und die narrative Dynamisierung dieser Räume erlauben zuweilen eine Erneuerung des Topos und seiner semantischen Besetzung, d. h. letztlich des Mythos. Dabei eröffnen die beiden Filme Gladiator und Rome, so meine Argumentation, auch eine je andere Perspektive auf die historischen Ereignisse und entfalten verschiedene filmische Geschichtsbilder.

\section{Die Stadt Rom als ikonographischer Topos und Gedächtnisbild}

Auch wenn Historienfilme ein relativ stabiles Geschichtsbild vom antiken Rom evozieren, ist diese wertmäßig-emotional aufgeladene Ikonographie nicht zeitlos: Vor dem Hintergrund sich wandelnder Produktionsbedingungen, technischer Entwicklungen und gesellschaftlicher wie diskursiver Kontexte besitzt jeder Film - jeder Roman, jedes Gemälde - vielfältige Möglichkeiten, dem To-

2 Barthes (1957) 191-247. 
pos eine neue Facette hinzuzufügen, ihn durch ästhetische und narrative Entscheidungen zu modifizieren. Verstehen wir Traditionsbildung mit Paul Ricœur als sich selbst strukturierende ,kumulative` Dynamik, die im Verhältnis von Muster und Einzelfall Veränderung zulässt, so können wir von einer ,Sedimentierung der Stadtbilder von Rom im kulturellen Gedächtnis ausgehen. ${ }^{3}$ Ähnlich einem Palimpsest scheinen die früheren Entwürfe in jeder Aktualisierung durch oder werden implizit durch Abweichung aufgerufen. Diese transhistorische, transtextuelle und transmediale Bezugnahme basiert auf Wiederaufnahme und Umformung des Vorgängigen nach dem semiotischen Modell von ,Repetition und Innovation', das Umberto Eco vorgelegt hat. ${ }^{4}$

Jeder Film ,erneuert‘ auf diese Weise die in unserer westlichen Kultur sedimentierten Erinnerungsbilder der antiken Stadt Rom. Der Prozess ihrer Aneignung kann mit dem der Geschichtserfahrung verglichen werden, für die Walter Benjamin den Begriff des ,dialektischen Bildes“ eingeführt hat, als einer imaginären Konstruktion von Geschichte, eines ,Gedächtnisbildes‘, in dem Gegenwart und Vergangenheit sich miteinander verbinden und gegenseitig durchdringen: ${ }^{5}$ „Das dialektische Bild ist ein aufblitzendes. So, als ein im Jetzt der Erkennbarkeit aufblitzendes Bild, ist das des Gewesenen [...] festzuhalten."6 Auch wenn in der geschichtsphilosophischen Perspektive Benjamins die Frage nach dem Vergangenen dominiert, das nur als Bild und nur als Bild der Erinnerung in der Gegenwart überhaupt ,aufblitzt ${ }^{\text {} ~ o d e r ~ a r t i k u l i e r b a r ~ i s t, ~}{ }^{7}$ so ist die Frage nach der Neuerung und Veränderung topischer Bilder - kraft der Dialektik - in seinem Konzept enthalten. Begreift man mit Jörg Schweinitz Topoi als „evolutionär[e] offen strukturiert[e] Netzwerk[e] von Stereotypen“,8 sind diese einem steten Wandel unterworfen: Jede aktualisierende Praxis des Topos der antiken Stadt Rom, der sich im Film aus ikonographischen, narrativen und generischen Elementen zusammensetzt, bedient das Gedächtnisbild und verschiebt es - manchmal kaum merklich, sodass wir nur die Wiederholung erkennen und negativ von Klischees sprechen; manchmal ist die Abweichung hingegen wahrnehmbar, und der Umgang mit dem diffus in unseren Köpfen existierenden Muster wird als originell und kreativ empfunden oder zumindest als Differenz erkannt.

3 Ricœur (1984) 30-40, hier 31.

4 Eco (1985). Zu den verschiedenen transtextuellen Modi der Beziehung zwischen ,Hypotext ${ }^{\star}$ und ,Hypertext‘ vgl. Genette (1982) 7-17.

5 Zu Benjamins Auffassung der Geschichte als ,Gedächtnisbild‘ vgl. Schöttker (2004) 25-27.

6 Benjamin (1977a) 245.

7 Vgl. auch Benjamin (1977b) 253.

8 Schweinitz (2006) 86. Der Autor spricht an dieser Stelle von (filmischen) Genremustern als ,topischen Systemen‘. 


\section{Prozesse der Semantisierung und Diskursivierung der Stadt}

Solchermaßen zwischen Wiederholung und Verschiebung oszillierende Topoi transportieren und kreieren kulturelle Werte. Durch die Auswahl von Orten, die Ausstattung von Räumen und deren filmische Gestaltung ist die Ikonographie des antiken Rom immer schon mythisch, das heißt nach Roland Barthes ideologisch oder zumindest axiologisch besetzt, selbst wenn ihre Elemente durch Abnutzung ,naturalisiert' oder ,entpolitisiert' wurden. ${ }^{9}$ Dennoch figurieren Paläste, die curia, wo der Senat tagt, das Kolosseum etc. als architektonische Repräsentationen der Macht auf der Inhalts- wie auf der Ausdrucksebene eines Films. Umgekehrt wird das Bild der Stadt auch durch die Auslassung von bestimmten Orten und Räumen sozusagen ex negativo mit Bedeutung aufgeladen. Dabei sind etwa Lebens- und Handlungsräume von Sklaven im Antikefilm generell unterrepräsentiert, denn diese dienen - von den zahlreichen Spartakus-Filmen einmal abgesehen - meist nur als Dekorfiguren. ${ }^{10}$ Die Serie Rome, die der Ausgestaltung von Alltagssituationen allgemein viel Platz einräumt, schafft diesbezüglich neue Bilder; auch die ,neuen Bilder sind selbstverständlich nicht neutral, doch sie können sich in die Lücken des Gedächtnisbildes der antiken Stadt einschreiben und dieses ergänzen. ${ }^{11}$ Außerdem ist dieser durch Bilder und Töne geschaffene diegetische Raum im Film von vornherein immer in Bewegung, und bereits durch ein originelles Requisit, eine kleine Geste, eine atmosphärische Lichtführung können die Elemente des Topos in Fluss geraten, das Stadtbild neu beleben und partiell resemantisieren. Die fiktionale Welt oder Diegese eines jeden Antikefilms setzt sich so aus tradierten ikonographischen Motiven, die mehr oder minder bekannt und abgenutzt sind, und originellen oder zumindest weniger vertrauten Elementen zusammen.

Diese erste Ebene der (Re-)Semantisierung von imaginären Stadträumen erfährt zudem eine Dynamisierung durch die filmische Narration. Im audiovisuellen Erzählprozess, der die Figurenhierarchie sowie die Verkettung der einzelnen Szenen und Handlungsschauplätze einschließt und eine Perspektive auf den Fortgang des Geschehens vermittelt, können dem städtischen Raum unterschiedliche Funktionen zukommen. Tritt sie aus ihrem Status als amorphes Dekor im Hintergrund des Geschehens heraus, so wird die Stadt als Gan-

9 Barthes (1957) 229-233.

10 Zur filmischen Darstellung von Sklaven allgemein vgl. Eigler (2005) und Wieber (2012); zur Figur des Spartakus vgl. ausführlich Späth / Tröhler (2008).

11 Vgl. Späth (2010). 
zes oder einzelne ihrer Räume und Bauten zum bestimmenden Ort für Figuren, emotionale Situationen und Handlungsmomente sowie für deren Verknüpfung. Während nun Gladiator den städtischen Raum mittels technischer Neuerungen zur exzessiven Inszenierung einer Architektur von Macht und Gegenmacht nutzt, um das Protagonistenpaar des Helden und des Antagonisten zu charakterisieren, lässt Rome durch seine plurale Figurenkonstellation, die auch eine Verlagerung der klassischen narrativen Perspektive zur Folge hat, polyphone Räume entstehen und eröffnet eine dezentrierte Sicht auf die (historischen) Ereignisse.

Der imaginäre Raum der Stadt Rom erfährt im Prozess der Narrativierung in beiden nachfolgend näher besprochenen Filmproduktionen jedoch nicht nur eine weitere Semantisierung. Vielmehr lässt sich in der unterschiedlichen Verräumlichung der Narration auch eine Metaposition herauslesen, durch die der audiovisuelle Diskurs auf seine Historizität aufmerksam macht und selbstreflexiv Stellung bezieht zur eigenen Repräsentation von Geschichte. ${ }^{12}$ Im Rahmen ihrer jeweiligen Unterhaltungsmodelle und -formate machen die beiden Filme zwei historiographische Konzeptionen deutlich: In der urbanen Topographie von Gladiator kristallisiert sich über die monumentale Bildlichkeit und das mythische Erzählmodell des Films die klassische Auffassung einer Personen- und Ereignisgeschichte heraus, während die städtischen Räume und Orte in Rome die dehierarchisierte Perspektive einer Alltags- und Sozialgeschichte reflektieren. Obwohl beide Filme mit ihren Weltentwürfen einen innovativen Beitrag zur narrativen, populären ,Geschichtsschreibung‘ liefern, meißeln sie auf je unterschiedliche Weise am Topos des sedimentierten Bilds der Antike. ${ }^{13}$

\section{Pompöse Räume der Macht}

Triumphzüge gehören zu den medialen ,Schlüsselbildern“, die die Antikefilme von der Stadt Rom in das kulturelle Gedächtnis eingegraben haben. ${ }^{14}$ Ein Ver-

12 Vgl. Rosen (2001) XII-XVIII; 353-354.

13 Ob Film - als Medium und innerhalb der Mainstreamkonventionen und der ökonomischen Zwänge der Filmindustrie - als der wissenschaftlichen, schriftlichen Historiographie ebenbürtig betrachtet werden kann, steht hier nicht zu Debatte; ebensowenig wie die Frage nach der historischen Authentizität - oder im Gegenzug dazu den Anachronismen - der gestalteten Welten und Ereignisse. Aspekte zu dieser Diskussion sowie zum Verhältnis von Wissensvermittlung und Unterhaltungswert, das bereits in der antiken Geschichtsschreibung verhandelt wird, bieten u. a. Rosenstone (1995); Tatum (2008) oder Späth / Tröhler (2012) insbesondere 269-271; 288-292.

14 Ludes (2001). 
gleich der entsprechenden Sequenzen aus beiden Filmen bietet sich somit als Einstieg an, um deren audiovisuelle Gestaltung und narrative Einbettung sowie die Funktion von urbanen Räumen für das entworfene Geschichtsbild zu analysieren.

Die Handlung von Gladiator situiert sich zur Zeit des Kaisers Commodus um 180-192 n. Chr. Commodus ist der Sohn von Mark Aurel, der sich im Film gegen den Willen seines Vaters die Macht aneignet; er wird als Alleinherrscher gezeigt, der eine korrupte Politik betreibt. ${ }^{15}$ Gegen ihn steht die fiktive Figur Maximus, ein General, der von Mark Aurel als legitimer Nachfolger bestimmt, jedoch von Commodus als Konkurrent ausgeschaltet, versklavt und in die Verbannung geschickt wird. Als Gladiator kommt er nach Rom zurück und kämpft in der Arena selbstlos für die Wiederherstellung der senatorischen Republik doch davon später mehr.

In der zur Debatte stehenden Sequenz zieht Commodus als Imperator mit seiner Schwester Lucilla nach der (von Maximus) gewonnen Schlacht und dem Tod seines Vaters in Germanien in einem monumentalen Triumphzug in Rom ein. Die Stadt erscheint hier - nach etwa einer Stunde des Films - zum ersten Mal: Die computergenerierten Bilder in der Totalen simulieren eine Flugaufnahme auf das dichte Geflecht der Straßen von Rom und seiner Repräsentationsbauten. Für diese Gesamtansicht diente Mussolinis antikes Stadtmodell, das im Rahmen der Mostra Augustea 1937 ausgestellt wurde, als Vorlage. ${ }^{16}$ Die annähernd monochrom in Grau gehaltene Farbgestaltung zieht sich als Dominante durch die ganze Sequenz. Eine Aufsicht zeigt das kaiserliche Gespann auf der Paradestraße, gerahmt von den geordneten Reihen der Legionäre; im Hintergrund ragt das Kolosseum auf. In einer schnellen Folge ineinander überblendeter Einstellungen sehen wir - nun auf Augenhöhe der Figuren - Ausschnitte aus der anonymen Masse unzufriedener Bürger, die durch Soldaten in Schach gehalten werden. Überlebensgroße Statuen und omnipräsente Adlerstandarten säumen den Weg von Commodus und Lucilla durch den Triumphbogen zum Senatsgebäude, wo sie von Blumenkindern, darunter Lucius, dem Sohn von Lucilla, und einigen Senatoren empfangen werden. Bevor der Dialog und damit die Handlung wieder einsetzt, markiert eine synthetische Einstellung auf die saubere Massenchoreographie und die herannahende Quadriga den Abschluss der spektakulären Bilderfolge des Triumphzugs (Abb. 1).

15 Der Film von Ridley Scott ist ein (uneingestandenes) Remake von The Fall of the Roman Empire (Anthony Mann, USA 1964); zum Vergleich der beiden Filme siehe Winkler (2009) 21-25.

16 Vgl. Stähli (2008) 115. 


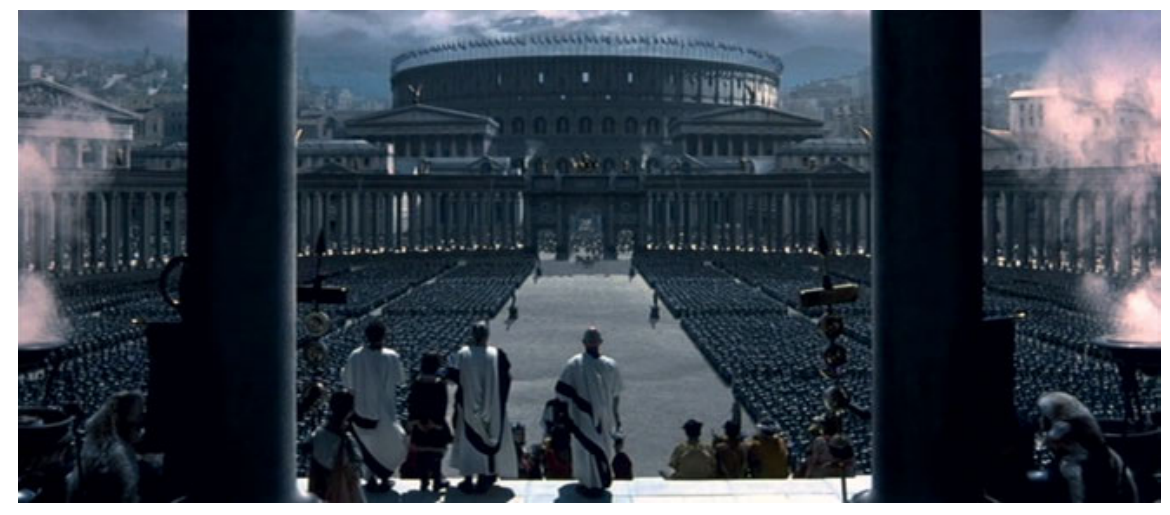

Abb. 1: Virtuelle Massenchoreographie des Triumphzugs in Gladiator (Ridley Scott, USA 2000).

All diese Elemente charakterisieren die Stadt Rom dem Bild entsprechend, das der Film von dem Tyrannen Commodus in der Rolle des Antagonisten entwirft: ${ }^{17}$ als Raum der Macht, der Kontrolle, des Terrors. Für diese totalitär konnotierte Inszenierung hat sich Ridley Scott bekanntermaßen an Leni Riefenstahls Triumph des Willens (D 1935) und dessen faschistische Ikonographie des Nürnberger Parteitags von 1934 angelehnt. Auf diese transtextuelle Semantisierung des Stadtraumes in Gladiator haben unter anderem Arthur Pomeroy oder Adrian Stähli hingewiesen. ${ }^{18}$ Zwar ist die Inszenierung des Triumphzuges durch dessen narrative Rahmung und Assoziation mit der tyrannischen Herrschaft von Commodus hier eindeutig negativ besetzt, die bildgewaltige Attraktion dieser Sequenz hat jedoch einen ähnlich betörenden Effekt wie bei Riefenstahl. ${ }^{19}$ Außerdem wurde die moderne digitale Technologie, die hier zum Einsatz kam, durchwegs positiv als Spektakelwert des Films beworben und besprochen.

Eine zweite Sequenz, die auf die an den Triumphzug anschließende Konfrontation zwischen Commodus und dem Senat und die Auseinandersetzung zwischen Commodus und Lucilla über dessen Herrschaftsvision folgt, verdeutlicht - wiederum über die Rauminszenierung - die menschenverachtende Politik des Imperators, der das Volk mit einem Überangebot an Spielen für sich zu gewinnen und zufriedenzustellen hofft. Sie bestätigt aber auch die ästhetische

17 Zum Begriffsfeld des Antagonisten vgl. Wulff 2002.

18 Vgl. Pomeroy (2004) 116; Stähli (2008) 115 f.

19 Vgl. Stähli (2008) 100-101. 


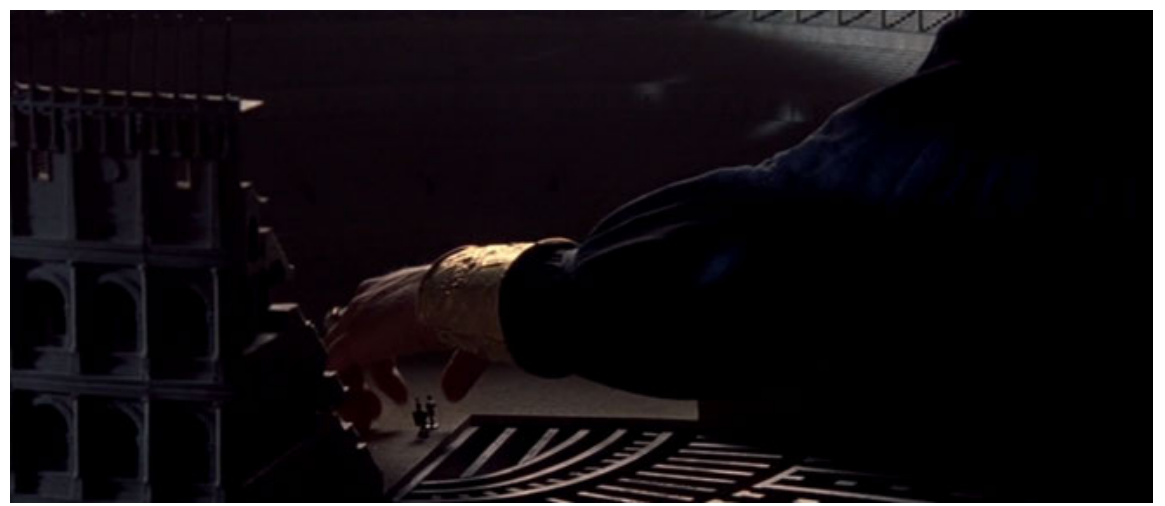

Abb. 2: Gladiator: Das Kolosseum als Modell für die Machtspiele von Commodus.

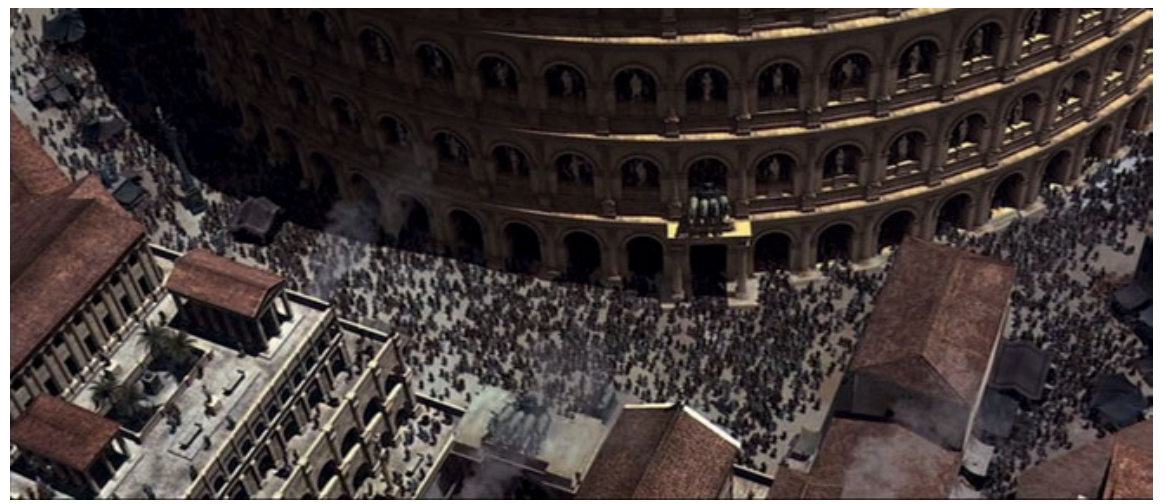

Abb. 3: Gladiator: Via sacra und Kolosseum als virtuelle Rekonstruktion.

Konzeption des Films hinsichtlich seiner monumentalen, auf Institutionen fokussierten Ikonographie sowie seine narrative Zentrierung auf einen einzigen Handlungsstrang.

Die Hand des Imperators manipuliert in einer Nahaufnahme die Gladiatoren als Spielfiguren in der Arena des Kolosseumsmodells (Abb. 2). Dieses nachgebaute Modell ist nicht nur ein synekdochisches Sinnbild von Commodus' Macht, sondern auf diegetischer wie auf ästhetisch-technischer Ebene auch eine Mise en abyme: In einer erneut einsetzenden digitalen Flugaufnahme gleitet der Blick sodann über die modellhafte Via sacra zum Kolosseum (Abb. 3) und schreibt die moderne Technologie, begleitet von bombastischen Musikklängen und dem Geschrei der Masse, weiter in einen politisch zumindest zwiespältigen Diskurs ein. Ein kontrastierender Schnitt bringt einen Szenen- 


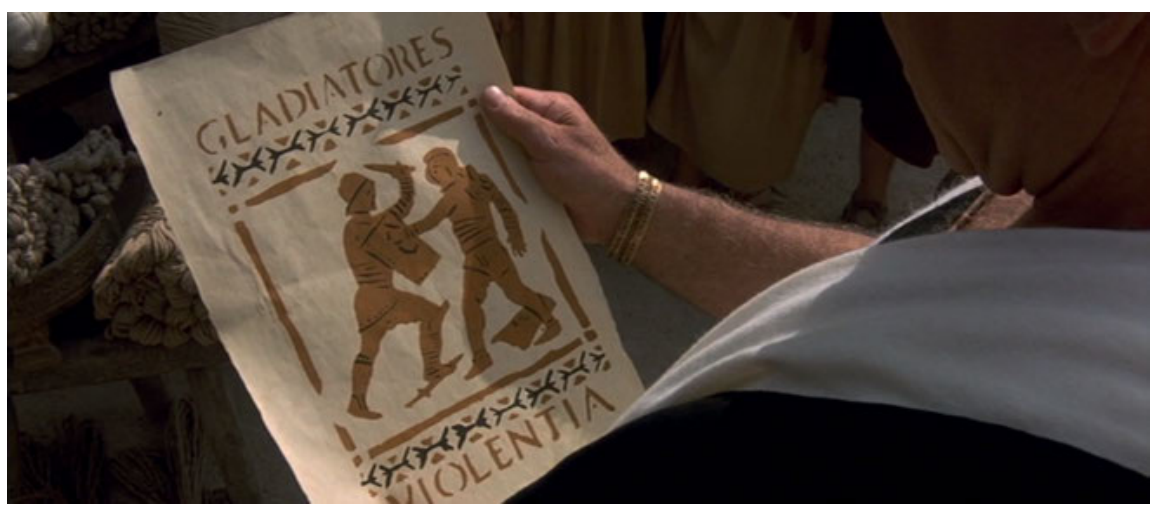

Abb. 4: Gladiator: Ein Flugblatt kündigt die Gladiatorenspiele an.

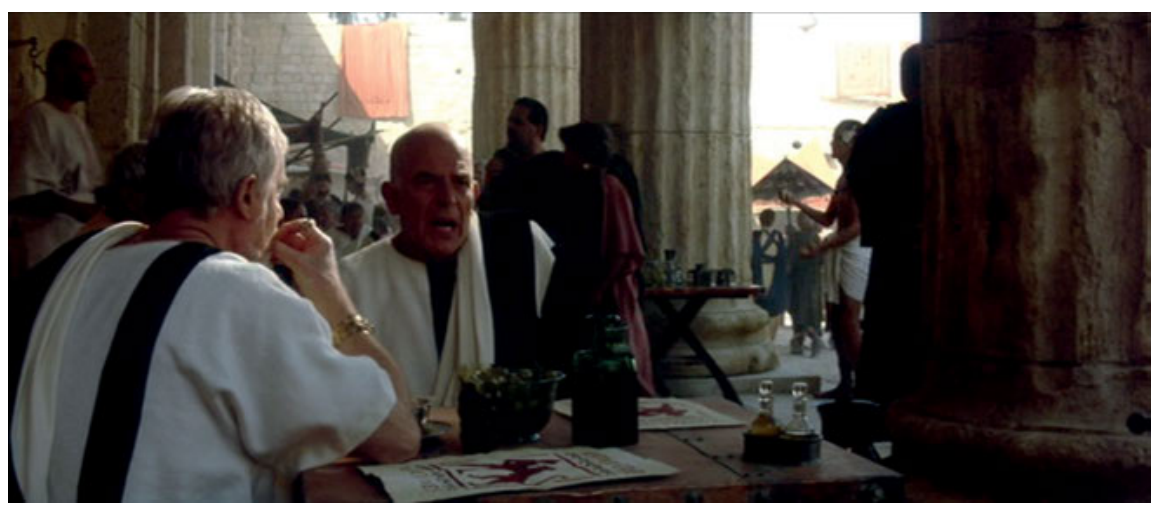

Abb. 5: Gladiator: Das urbane Alltagsleben als Dekor im Hintergrund.

wechsel: Eine Großaufnahme zeigt ein Flugblatt, das Gladiatorenkämpfe ankündigt und den Handlungsfaden wieder aufnimmt (Abb. 4): Der Senator Gaius, der das Flugblatt in den Händen hält, begibt sich durch die Straßen zum Treffen mit seinem Kollegen Gracchus. Während die beiden über die Funktion der Spiele für die korrupte Politik von Commodus sprechen, sehen wir im Hintergrund Passanten, einen Jongleur, asiatisch anmutende Sonnenschirme (Abb. 5). Die überwiegend als Nahaufnahmen gefassten Einstellungen lassen durch ihre Konzentration auf den Dialog zwischen den beiden Senatoren indes nur knapp das Dekor einer Taverne unter den Arkaden erahnen.

Die Straßen und die Bevölkerung Roms sind auch in dieser Sequenz entweder nur als Elemente einer Ikonographie der Monumentalität und der Masse präsent, oder das kaum differenzierte Menschengewimmel und der Straßen- 
lärm gehören zur atmosphärischen Ausstattung eines anonymen Stadtraums im Hintergrund der ereigniszentrierten Handlung, die durch das (hier nun im narrativen Sinne) mythische Muster eines antagonistischen Paars, den Kampf zweier Titanen, bestimmt ist.

So gibt es in diesem Film kaum relevante Nebengeschichten, es ist kein Platz für städtische Lebensräume und einen sozialen Alltag: Die audiovisuelle Gestaltung der urbanen Topographie konzentriert sich zunächst auf die durch den Imperator personalisierten Institutionen der Macht: Paradestraßen, Triumphbögen, den Palast und die Gemächer des Kaisers, den Versammlungsraum des Senats, die curia. Im zweiten Teil des Films verlagert sich die Aufmerksamkeit sodann auf die Arena mit ihrem manipulierbaren Mob und auf die Substrukturen des Kolosseums. Dieses dient dabei als Ort der Herausforderung des Imperators durch Maximus, den eigentlichen Helden des Films, und als Hort der Gegenmacht, wo im Untergrund das Komplott gegen Commodus angezettelt wird. ${ }^{20}$

Sicher ist, dass die Stadt in diesem Film insbesondere dank der digital generierten und diegetisch-filmisch inszenierten Monumentalität ihrer Architektur mehr als nur eine Dekorfunktion besitzt. Ikonographisch fügt sich so dem topischen Gedächtnisbild der Antike eine neue Facette hinzu. Der technische Exzess, für den Gladiator in die Filmgeschichte eingegangen ist, macht auch selbstreflexiv auf sich aufmerksam, jedoch erscheint die Metaposition, die der Film zum Entwurf seines Geschichtsbildes einnimmt, ambivalent. Auf ikonographischer Ebene sind die Stadtansichten mit ihren Repräsentationsbauten und symbolischen Orten der Machtausübung durch den transtextuellen Echoraum zunächst negativ besetzt. Diese Deutung wird durch die auf narrativer Ebene angelegte Distanzierung von Commodus' imperialem Größenwahn, in den sich die Dekadenz und damit der ,Fall' seiner Herrschaft von Anfang an einschreiben, gestützt. Das audiovisuelle ,Spektakel triumphiert indes über die semantische Tiefe der aufgerufenen Referenz und deren kulturelle Wertung. Durch den pompösen Einsatz der Technik, die Bildkompositionen und die filmische Mise en scène kann der Raum der antiken Stadt und der Macht wohl ,körperlich und konkret' und damit sinnlich erfahren werden: „New Technology brings History to Life“. ${ }^{21}$ Doch um mit Fredric Jameson zu sprechen: Der Stilpastiche, der die brillante Oberfläche als ,Intensitäten' feiert und die Materialität der Bilder technisch und ästhetisch als Warencharakter ausstellt,

20 Die Räume außerhalb der Stadt Rom, das Schlachtfeld, das Feldherrenzelt, die Gladiatorenschule etc. bestätigen dieses Bild, sind hier aber nicht von Belang.

21 Dies ist der Titel eines Textes, der die neue Technologie in Gladiator preist, vgl. http:// web.uconn.edu/english294/gladiator.html (30. Juli 2013). 
verwehrt den Bildern ihre historische Dimension und eine bewusstseinsmäßige Aufarbeitung. Durch den postmodernen Umgang mit den Elementen des Topos der korrupten Macht (in Bezug auf die Antike wie den Faschismus) wird die „Vergangenheit als ,Referent“ [...] in Klammern“gesetzt. ${ }^{22}$

\section{Horizontale Verräumlichung von Macht}

Wenden wir uns nun der Verräumlichung der antiken Stadt in der TV-Produktion Rome von HBO und BBC zu, die durch das Format der Serie mit (in diesem Fall) zwei Staffeln und insgesamt 22 Episoden gewiss ganz andere Voraussetzungen genießt als ein zweieinhalbstündiger Kinofilm. Die Unterschiede, die in der Inszenierung des Triumphzugs auszumachen sind, lassen sich jedoch kaum auf ökonomische oder institutionelle Zwänge der beiden Industrien reduzieren: Sie zeugen von einer anderen Konzeption von Unterhaltung, von filmischer Attraktion und historischem Erzählen, in deren ästhetischer und narrativer Umsetzung hinsichtlich des urbanen Raumes auch ein anderes Geschichtsbild durchscheint. Die erste Staffel von Rome, auf die sich die vorgeschlagene Analyse beschränkt, spielt in der Zeit von 52 bis 44 v. Chr., sie beginnt mit Caesars Sieg über Vercingetorix in Alesia (Gallien) und endet mit seiner Ermordung in Rom. Der Triumphzug findet im Jahr 46 und hier in der 10. Episode (von 12 in der ersten Staffel) statt. Da eine Quality Soap wie Rome sich durch komplexe Erzählstrukturen und mehrere parallel verlaufende Geschichtenstränge - das bedeutet auch eine plurale Figurenkonstellation - auszeichnet, ${ }^{23}$ dauert der gesamte Triumphzug von den Vorbereitungen bis zu den Aufräumarbeiten rund 15 Minuten: Mehrmals schieben sich Parallelhandlungen, die sich an anderen Schauplätzen in der Stadt abspielen und nicht direkt kausal mit dem Triumphzug verflochten sind, dazwischen.

Nach diversen Vorbereitungen - Sklaven reinigen die Statuen (Abb. 6) und schmücken die Gebäude, vor den Toren der Stadt kleiden sich Soldaten festlich ein, Caesar diskutiert mit seinem Sklaven Posca und Marcus Antonius die Wahl seines Gewands, danach trägt Octavian die zeremonielle ockerrote Farbe auf sein Gesicht auf: die Farbe Jupiters und des Sieges (Abb. 7) - kann der Tri-

22 Jameson (1993) 58; 60; 63.

$23 \mathrm{Zu}$ den komplexen Erzählstrukturen von Quality Soaps vgl. Mittell (2006) oder Blanchet u. a. (2011); allgemein zu den Funktionsweisen mosaikartiger Figurenkonstellationen im Film vgl. Tröhler (2007) 385-526; insbesondere zum Geflecht von historischen und fiktiven Figuren in Rome vgl. Späth / Tröhler (2012) 276-288. 


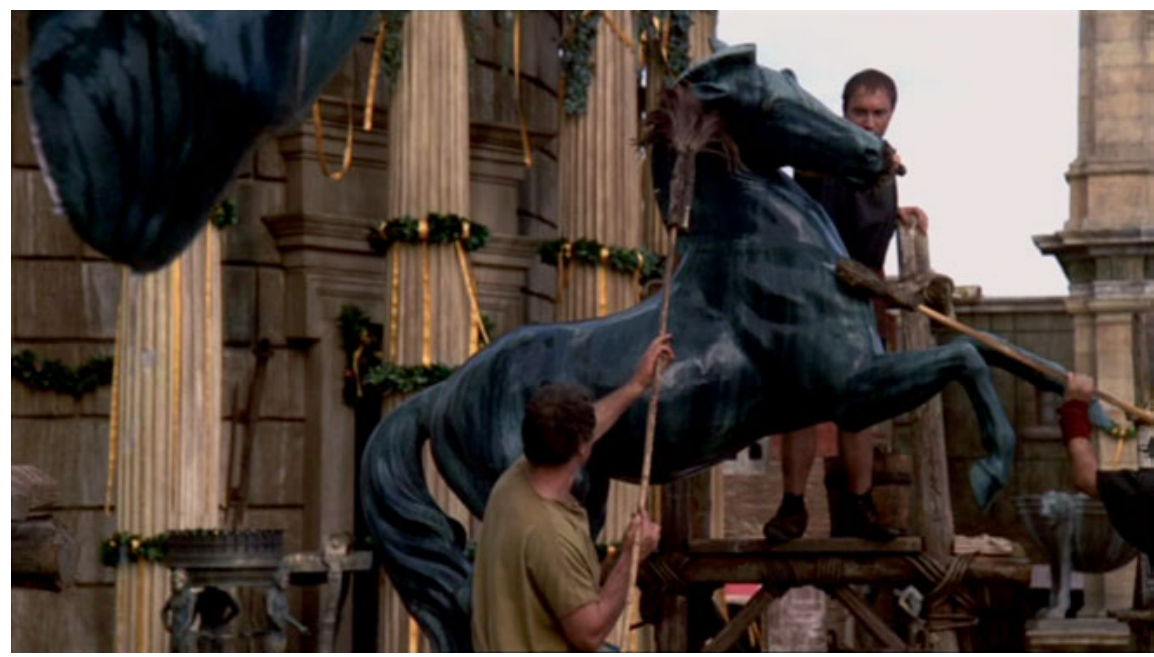

Abb. 6: Bilder des Triumphzugs in Rome (HBO/BBC, 1. Staffel 2005): Soldaten reinigen die Statuen.

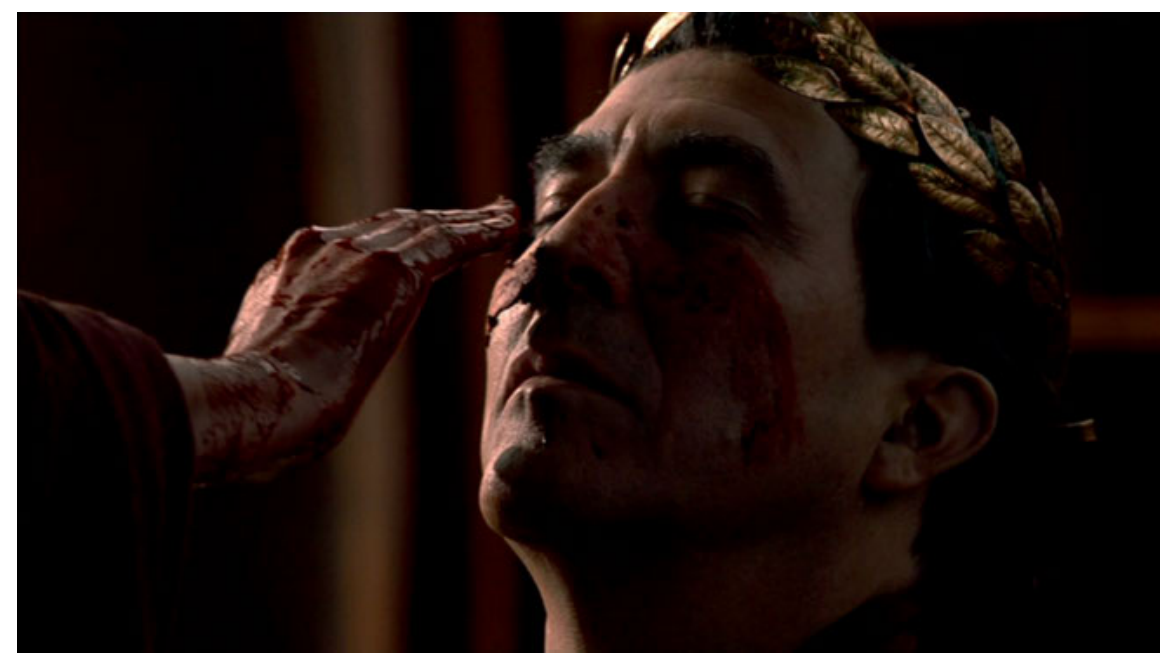

Abb. 7: Rome: Octavian bemalt Caesars Gesicht mit der roten Farbe des Triumphes.

umphzug beginnen. Das Ereignis setzt ohne viel Aufhebens und ohne Musik ein: Wir übernehmen als Zuschauer die optische Perspektive eines Kindes durch die Menschenansammlung, die nur ab und zu einen Blick auf den Umzug freigibt (Abb. 8). Sodann sind in der jubelnden Menge einige bekannte 


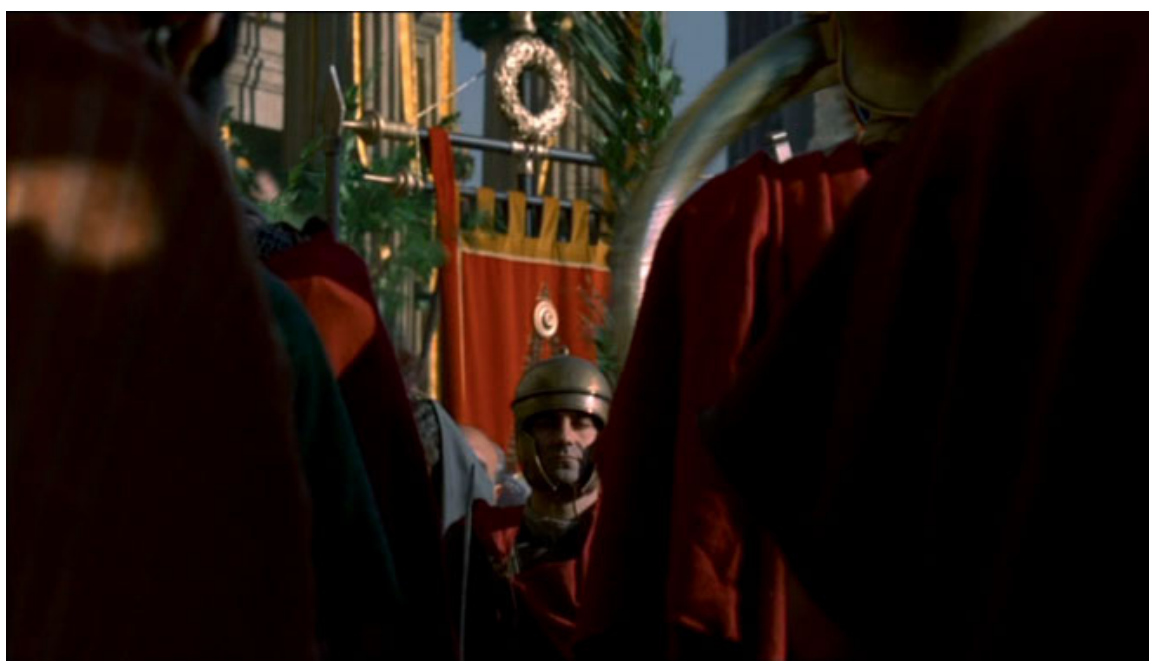

Abb. 8: Rome: Der Blick durch die Menge - auf Augenhöhe.

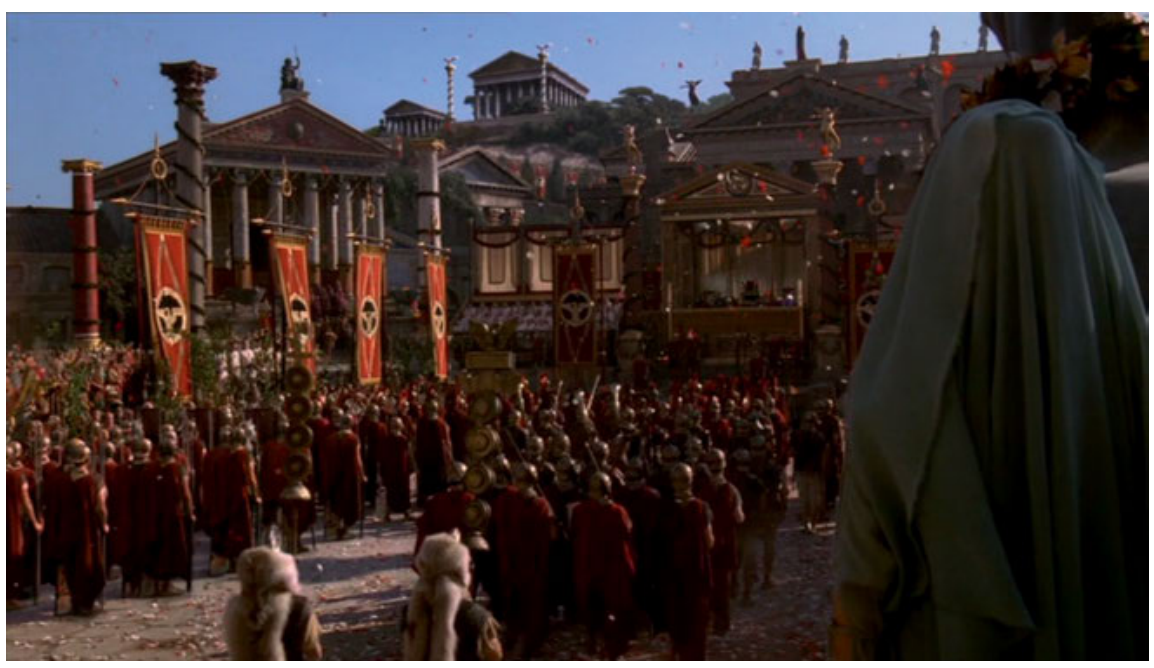

Abb. 9: Rome: Der durch eine Figur personalisierte Blick auf den Umzug und die Stadt.

Gesichter erkennbar, Niobe, die Frau und die Kinder von Vorenus sowie Vorenus selbst, der als Legionär und nun Leibwächter von Caesar sowie Senator von dessen Gnaden eine der Hauptrollen der Serie inne hat. Der Blick auf die Parade wird erneut durch die jüngere Tochter von Vorenus verkörpert und personalisiert. Jemand hebt sie im Laufe der Szene auf den Sockel einer Reiterstatue: Wir sehen sie im Bild und schauen mit ihr dem Triumphzug nach (Abb. 9). Der 


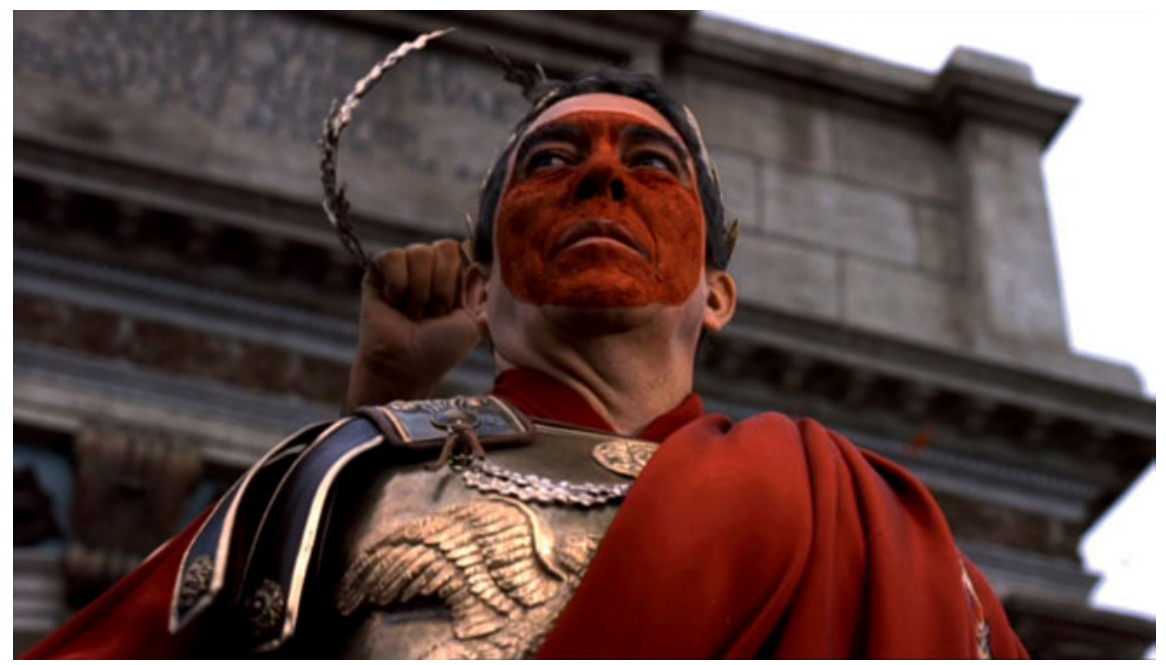

Abb. 10: Rome: Ein Sklave hält den Lorbeerkranz über Caesars Haupt; im Hintergrund der Triumphbogen.

Wechsel zu Caesar findet mit einer Aufnahme statt, die auch ihn von hinten zeigt, wie er auf seinem Wagen durch den Triumphbogen reitet (die Einstellung erweist sich kurz darauf als Blick des Sklaven, der den goldenen Lorbeerkranz über seinen Kopf hält; Abb. 10). Die Farben Rot, Weiß, Gold dominieren. Immer wieder wird die Aufmerksamkeit auf einzelne Figuren in der winkenden und staunenden Menge gelenkt. Auch auf der Tribüne sind uns als Zuschauer der Serie einige Figuren bekannt: Brutus, dem Cicero etwas ins Ohr flüstert - die Verschwörung gegen Caesar hat bereits begonnen -, und im vorderen Rang Atia, die Nichte von Caesar sowie deren Kinder, Octavia und Octavian, der spätere Augustus, ebenso Marcus Antonius, der in der Serie der Liebhaber von Atia ist, die so Politik macht: Sie werfen sich vielsagende Blicke zu respektive weichen dem Blick des anderen aus. Caesar nimmt in ihrer Mitte Platz.

Als er sich nach der öffentlichen Hinrichtung von Vercingetorix von der Menge bejubeln lässt, blicken wir mit ihm hinunter auf den Platz und die Reihen seiner Legionäre (Abb. 11): Diese Einstellung ist jener in Gladiator nicht unähnlich und gehört zur politischen Ikonographie von totalitärer Macht (auch außerhalb des Kinos). Sie soll hier jedoch ostentativ Macht dar- und ausstellen - die Frage der Repräsentation von Herrschaft wurde in der Ankleideszene zwischen Caesar, Posca und Marcus Antonius explizit thematisiert. Und sie steht im Kontrast zu der zuvor audiovisuell vermittelten sinnlichen Erfahrung des Triumphzugs auf Augenhöhe des Publikums - der Blick des Mädchens, 


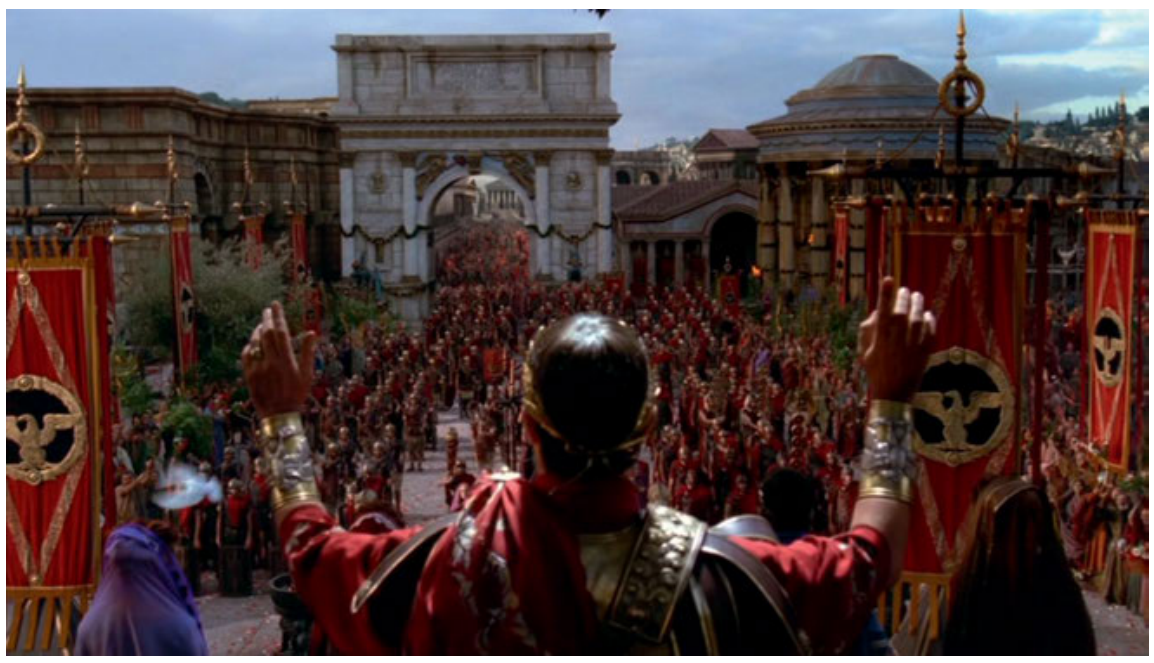

Abb. 11: Rome: Ausgestellte Ikonographie der Macht: Caesars Blick auf die Menge und das Forum.

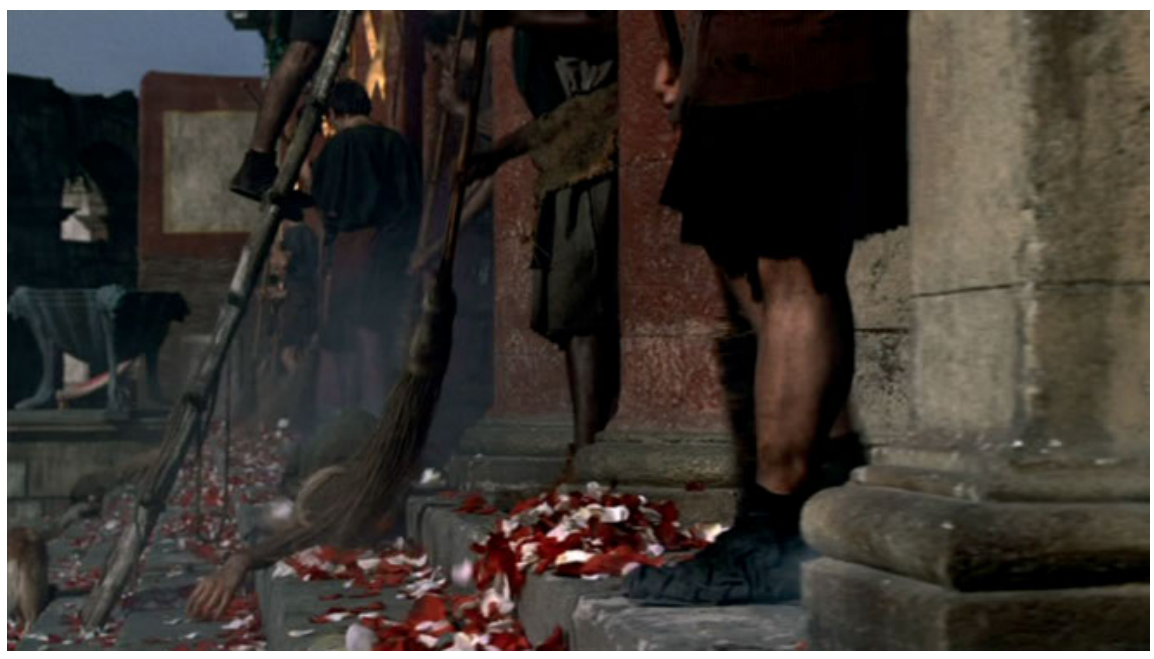

Abb. 12: Rome: Die Aufräumarbeiten am Morgen nach den Feierlichkeiten.

individualisierte Gesichter in der Menge, Detailaufnahmen auf Gesten und Requisiten. Diese ,gewöhnliche' Sicht auf das Ereignis rahmt die Machtdemonstration. Denn die Sequenz endet mit einem erneuten Kontrast, wenn nach einer Überblendung die Aufräumarbeiten und die Wiederaufnahme der alltäglichen Geschäfte in den Straßen Roms gezeigt werden (Abb. 12). 
Diese Sequenz ist für Rome aus mehreren Gründen außergewöhnlich: Weil sie sehr kostspielig und mit ihren 500 bis 600 Statisten sehr aufwendig war. Weil sie eine der raren Massenszenen der Serie enthält, die ansonsten statt auf Schlachtenbilder, Großanlässe und Menschenansammlungen auf Straßenbilder auf Augenhöhe und (dem TV-Bildschirm angepasste) kammerspielartige Szenen in Innenräumen setzt und eine Vielfalt von Milieus kontrastiert (aristokratische Villen und plebejische Stadtwohnungen, Innenhöfe, Tavernen, Bordelle und Feldzelte etc.). Und drittens: Weil sie einen dramaturgischen und historischen Wendepunkt andeutet: Caesar lässt sich mit diesem Triumphzug als definitiven Sieger feiern - auch über seine römischen Konkurrenten -, und in einer bestimmten historiographischen Perspektive (der seiner Gegner) maßt er sich mit diesem Akt den Status des Alleinherrschers an. Doch durch die wechselnde, polyphone Erzählperspektive erscheinen das (historische) Ereignis des Triumphzugs und Caesars Machtdemonstration audiovisuell in Alltagshandlungen eingebettet, durch Gesichter aus dem Volk kontextualisiert und räumlich in das dezentrierte Stadtbild eingelassen.

\section{Das verkörperlichte Bild des städtischen Alltags}

Was es bedeutet, die diegetische Welt der antiken Stadt Rom als polyphonen Lebens- und Verhandlungsraum zu gestalten, macht die Serie bereits in der allerersten Episode deutlich, wenn uns die Nebenfigur des Juden Timon - er ist Pferdehändler und Atias Mann für das Grobe, der sich seine Aufträge durch Liebesdienste bezahlen lässt - in die Stadt einführt: Die Kamera folgt ihm durch die belebten Straßen, begleitet von Alltagsgeräuschen und dem orientalisch (indisch) angehauchten musikalischen Leitmotiv der Serie (Abb. 13). Die Einstellungen nehmen am menschlichen Körper Maß (halbnahe bis halbtotale Einstellungsgrößen dominieren). Durch ihre Schärfentiefe ordnet sich die Figur von Timon in das Treiben ein, in welchem ihn die Kamera immer wieder aus dem Blick verliert. Diese filmische Gestaltungsweise macht aus dem urbanen Dekor einen Lebensraum mit seinen vielfältigen Handwerkstätigkeiten, sozialen Gesten, bunten Stoffen und einem ethnischen Gemisch von Menschen (Abb. 14 und 15). Wir begleiten Timon auch von einem Milieu zum anderen: von den engen Straßen eines populären Viertels über das Forum mit seinem Ausrufer hin zum ruhigeren, aristokratischen Villenquartier bis vor Atias Wohnhaus (Abb. 16).

Ob diese Weltgestaltung das antike Rom zur Zeit Caesars ,authentisch ' wiedergibt, sei dahingestellt; auf jeden Fall ist hier der städtische Raum mehr als 


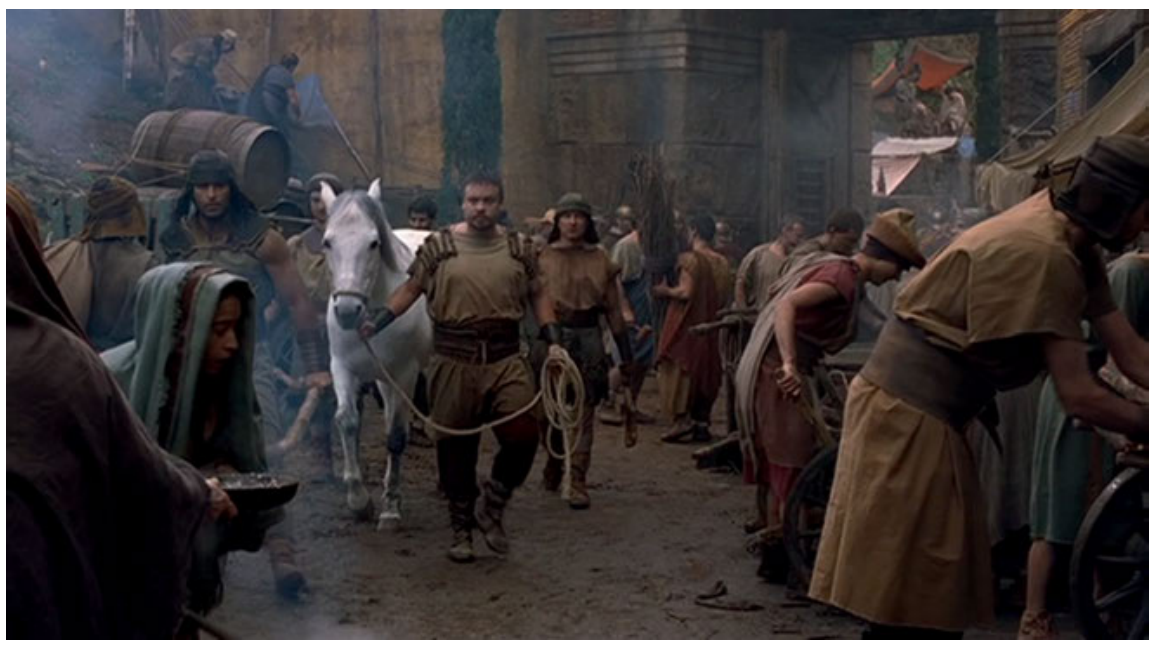

Abb. 13: Rome: Alltag in den Straßen Roms; in der Bildmitte Timon.

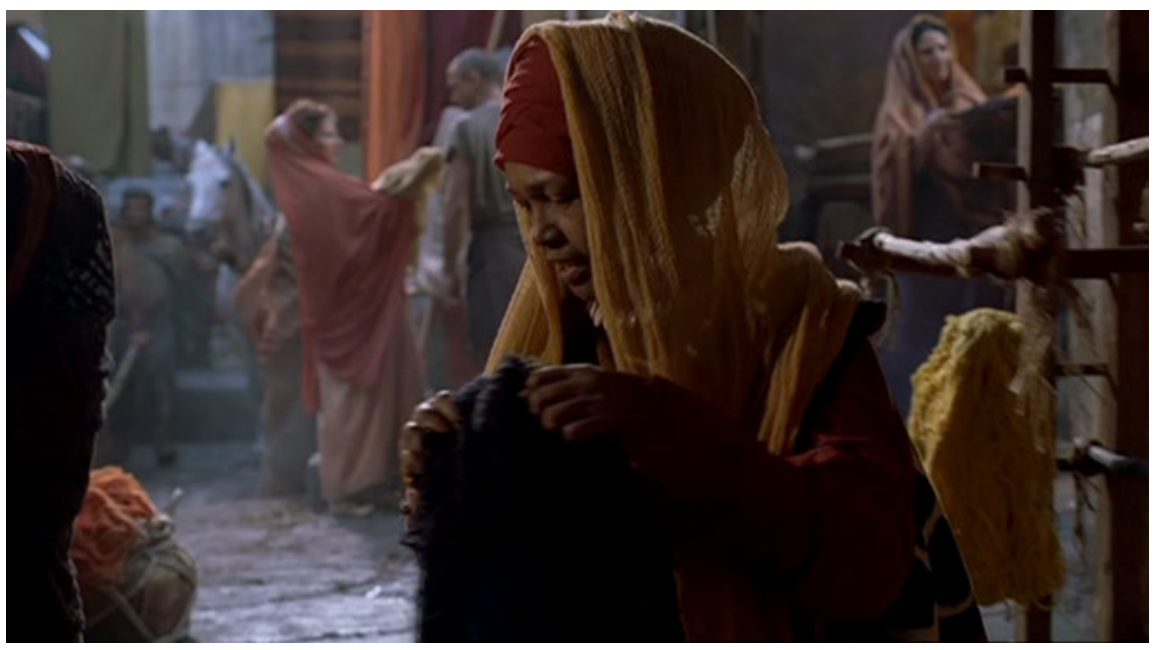

Abb. 14: Rome: Blicke in eine Färberei ...

atmosphärischer Hintergrund, und er ist auch nicht auf symbolische Orte der Machtrepräsentation reduziert. Vielmehr schafft die Serie eine bewegte und belebte diegetische Alltagswelt, in der die Aktivitäten der zahlreichen fiktiven und historischen Hauptfiguren motiviert sind.

Ikonographisch ergänzt und verschiebt Rome den Topos der antiken Stadt, fügt unserer Vorstellung von ihr eine sozusagen stoffliche Facette hinzu. Keine 


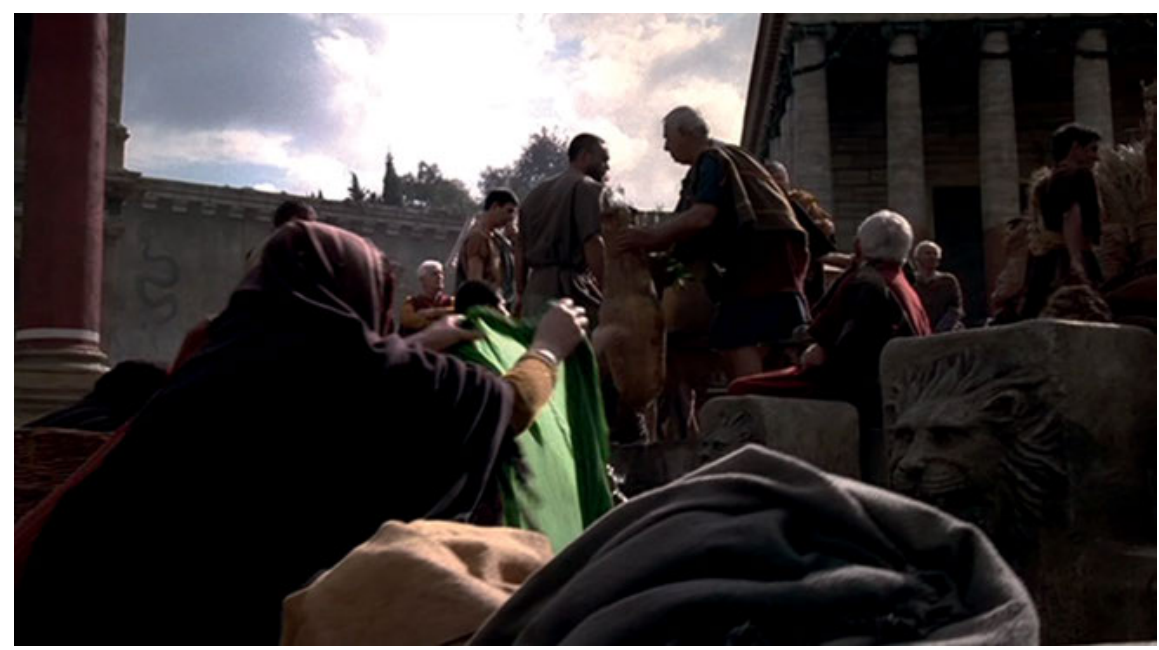

Abb. 15: Rome: ... und auf das bunte Treiben auf dem Forum.

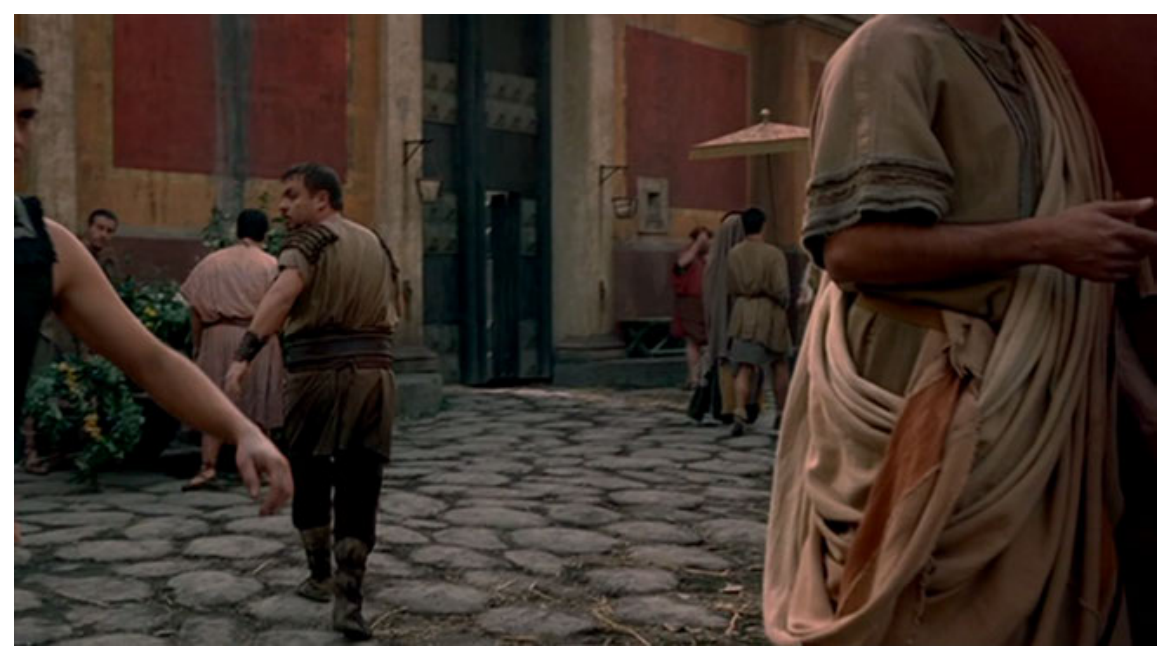

Abb. 16: Rome: Timon vor der aristokratischen Villa von Atia.

monochrome, weiße und saubere Marmorstadt mit ihren Institutionen der Gewaltherrschaft wie in Gladiator, keine völlig anonymen Massen, die dem narrativen historischen Geschehen nur als Mob und als Dekor dienen, d.h. als abstrakte Instanz; keine Totalen aus dem Himmel über Rom und keine digitale, technizistische Oberflächenästhetik - sondern: Bilder aus der Mitte der Stadt 
als Lebensraum, aus den bunten, staubigen Gassen, aus der sozial und ethnisch durchmischten Menschenmenge. ${ }^{24}$ Die Stadt stellt einen polyphonen personalisierten Raum mit ineinander verflochtenen Teilwelten oder Milieus dar. Unser Vorstellungsbild von Rom erweitert sich mittels der audiovisuellen Gestaltung sinnlich und semantisch: Die Stadt wird zu einem durch die Gesellschaft gebildeten ,Körper‘.

In ihrer narrativen Funktion sind die konkreten Orte und Räume - über die Charakterisierung der Figuren hinaus - auch Verhandlungsräume der sozialen und politischen Macht: Die Polyphonie der verschiedenen Alltags- und Lebenswelten, die sich durch eine ,Montage der voranschreitenden Gleichzeitigkeit" gegenseitig kommentieren, bestimmt die Erzähldynamik der Serie, die mit der Konzentration auf Einzelfiguren und Heldentaten bricht. ${ }^{25}$ Durch die Dezentrierung der Narration, die den Fokus abwechselnd auf verschiedene soziale Gruppen und ihre politischen Funktionen legt, wird eine ,stratifizierte Gesellschaft wie die römische erfahrbar. ${ }^{26}$ Die entsprechend verflachte Hierarchie zwischen historisch belegten und fiktiven Figuren aus allen Schichten verteilt die Handlungskompetenzen ,hypothetisch“ auf die gesamte Bevölkerung (respektive das gesamte Figurenarsenal) als Agens. So macht Rome „die Frage des [Stadt-]Raumes zur wichtigsten Problemstellung“ - oder zumindest $\mathrm{zu}$ einer der wichtigen - und erlaubt, um noch einmal Jameson zu zitieren, „ein Kartographieren der Wahrnehmung und der Erkenntnis“. Man könnte auch behaupten, dass die Serie Rome dem ,Historismus“ von Gladiator eine ,Historizität‘ entgegensetzt, indem sie „unser Vermögen, Geschichte aktiv und produktiv zu erfahren“, fördert. ${ }^{27}$ Die unterschiedlichen Erzählweisen der beiden Filme haben auch Auswirkungen auf die filmische Entwicklung des historischen Geschehens und das entworfene Geschichtsbild.

24 Die Serie wurde in Cinecittà gedreht; für die Inszenierung des bunten Treibens in der Stadt haben sich die Filmemacher nach eigenen Angaben die indische Stadt Mumbai (Bombay) zum Vorbild genommen; vgl. die ,Making-of'-DVD zur ersten Staffel.

25 Zur ,Montage der voranschreitenden Gleichzeitigkeit', die in mosaikartigen Erzählmustern das räumlich-horizontale Nebeneinander zahlreicher Schauplätze vor die zeitlich-kausale Handlungsverknüpfung stellt vgl. Tröhler (2007) 436-463.

26 Konzis zur hierarchischen römischen Gesellschaftsordnung Winterling (2004) 206.

27 Jameson (1993) 96 (Herv. i. O.); 66. Was Jameson in seinem 1984 zum ersten Mal erschienenen Aufsatz, in dem er seine tiefgreifende Kritik an der ,geschichtslosen' Postmoderne begründet, nur als dialektische Bewegung, als ,hypothetische' Wende ins Positive, formulieren kann, ist für Philip Rosens Verständnis einer ,radikalen Historizität' konstitutiv, welche durch die selbstreflexive Medialität der Filme eine historiographische Metaposition hervorbringen kann; vgl. Rosen (2006) 353. 


\section{Der mythische Kampf der Titanen vs. die Kontingenz von Geschichte}

Wenn in Gladiator Maximus als Superheld und Märtyrer dem Tyrannen Commodus gegenübertritt und ihn am Schluss in der Arena des Kolosseums herausfordert, soll er als Einzelner den Lauf der Geschichte verändern. Die Spannung zwischen den beiden Antagonisten bestimmt den Prozess der Narration. Dieses klassische Erzählmuster durchzieht den Film und prägt auch die Bildkomposition. Oft steht Maximus alleine und in Untersicht im Raum der Einstellung respektive der Arena, oder es wird auf den Zweikampf fokussiert (Abb. 17 und 18).

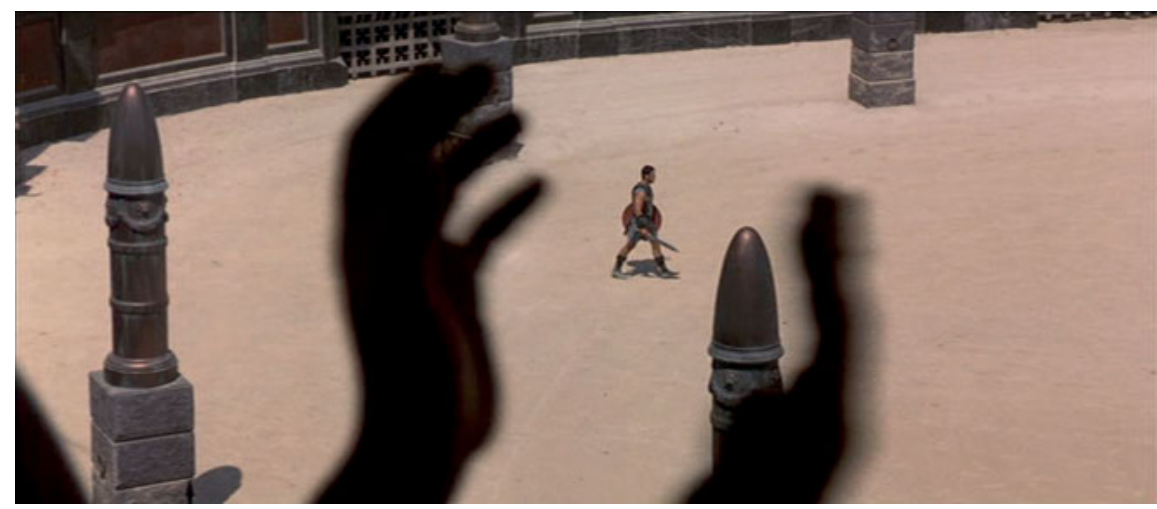

Abb. 17: Gladiator: Der Held Maximus alleine in der Arena: Ein Blick aus der Zuschauermenge.

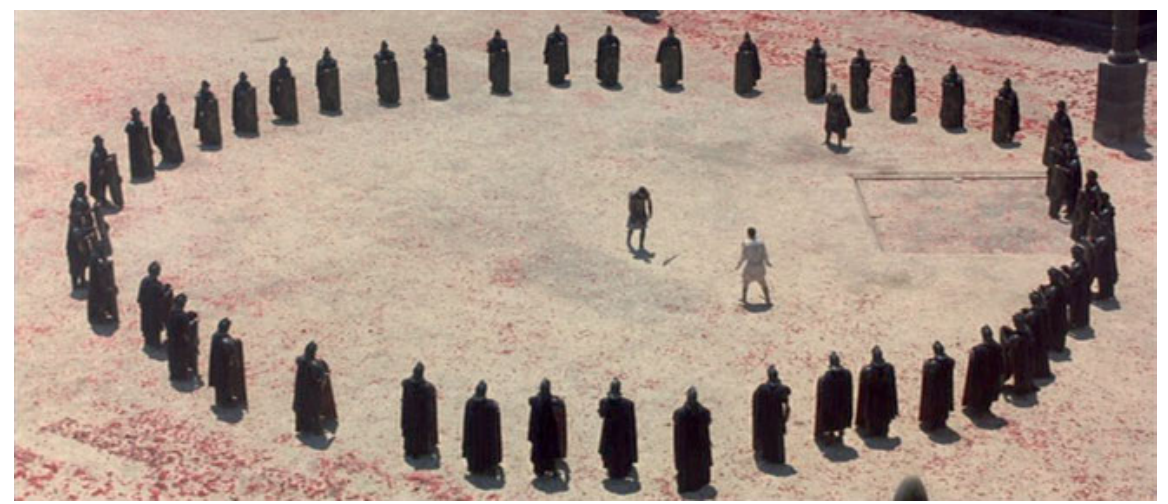

Abb. 18: Gladiator: Choreographie des finalen Zweikampfes zwischen Commodus und Maximus. 
Obwohl von einem Sklaven, also aus einer Position sozialer Erniedrigung kommend, gründet die politische Motivation von Maximus hauptsächlich in persönlichen Motiven der Rache und der Liebe. Politisch strebt er (um den letzten Willen Mark Aurels zu erfüllen) die Wiederherstellung der Republik an: Die Macht des Senats steht dabei für ein demokratisches Ideal. Einerseits übergeht der Film damit jedoch die Tatsache, dass die römische Republik ein oligarchisches Herrschaftssystem war; ${ }^{28}$ andererseits stellt er den imperialen Anspruch Roms (der sich auf die heutige USA übertragen lässt) nicht in Frage. ${ }^{29}$ Insgesamt scheint in Gladiator die neokonservative Haltung eines politischen Paternalismus durch - gekoppelt an die als universalistisch präsentierten gesellschaftlichen Strukturen und Werte von Klasse, Geschlecht, Familie, Moral und den Gegensatz von Stadt und Land. ${ }^{30}$ Die Mainstreamerzählung mit ihrer binären Dynamik, ihrer Konzentration auf einzelne Helden und monokausal hergeleitete Ereignisse trifft auf ein traditionelles Geschichtsbild, das die bestehende Ikonographie der Macht letztlich bestätigt, und auf die Eckpfeiler der klassischen Historiographie der Antike baut.

Im Gegensatz dazu entwirft die Serie Rome eine alternative Perspektive auf die antike Gesellschaft und Geschichte, indem sie soziale Räume und fiktive Figuren verschiedener Rangordnung auf die Entwicklung der historischen Ereignisse Einfluss nehmen lässt. Die urbane Topographie mit ihren Innen- und Außenräumen dient als Matrix für die Begegnungen und Situationen, in denen Macht innerhalb verschiedener Konflikt-,Felder` (als einem Zusammenwirken von ,Praktiken und Vorstellungen') ausgehandelt wird. ${ }^{31}$

Durch die polyphone, komplexe Erzählweise, die unzählige individuelle und doch voneinander abhängige Geschichten in einem Beziehungsnetz verknüpft, das sich durch den Stadtraum zieht, wird in der Interaktion zwischen Einzelfiguren und Gruppen auch die Trennung zwischen Gesellschaft und Politik aufgehoben. ${ }^{32}$ So ist es in der Serie sogar historischen Nebenfiguren und fiktiven Figuren aus sozialen Gruppen - wie Frauen, Sklaven, einfachen Leuten aus dem Volk -, die in der antiken Gesellschaft von der direkten Machtausübung ausgeschlossenen waren, möglich, den Verlauf des Geschehens, d.h.

28 Vgl. Pomeroy (2004) $121 \mathrm{f}$.

29 Vgl. Stähli (2008) 110.

30 Vgl. Pomeroy (2004) 123. Zur metaphorischen Übertragung der mythischen Semantik auf die Politik der USA schreibt z. B. auch Lee Weston Sabo (2012): „[...] the success of Ridley Scott's boneheaded remake, Gladiator, was due to the Republican fervor riding high during the 2000 election." (o. S.).

31 Bourdieu (1980) 88.

32 Vgl. Winterling (2001). 


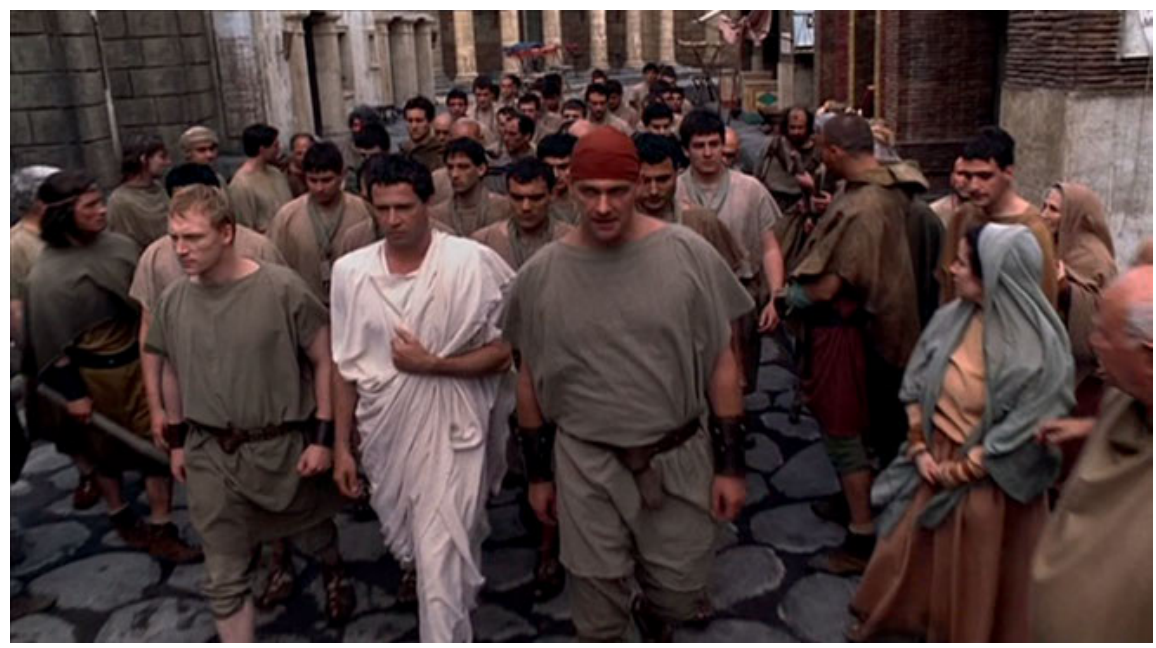

Abb. 19: Rome: Marcus Antonius, Pullo und Vorenus mit ihrem Gefolge auf dem Weg zum Senat.

auch die narrative Dynamik der Historie, zu verändern. Dadurch entsteht nicht eine revolutionäre Perspektive auf eine ,Geschichte von unten', sondern der Eindruck von Kontingenz durch alltägliche Praktiken, von wechselseitig sich bedingendem Handeln, das sich oft ohne Intention und konkrete Motivation als eine Konstellation von Möglichkeiten präsentiert - was Daniel Milo als Ausgangspunkt für eine ,experimentelle' Geschichtsschreibung postuliert, deren Aufgabe es sei, die Geschichte - gerade auch über die Narration - zu provozieren. ${ }^{33}$

Die Entwicklung eines Geschehens wird durch die verschachtelte Narration oft von langer Hand vorbereitet und zieht sich - vor dem Hintergrund der Stadt - durch die plurale Figurenkonstellation, die unterschiedlichsten Räume, Situationen und Milieus. Aus der zwischen mehreren Schauplätzen alternierenden Montage schält sich sodann ein Ereignismoment heraus, das komplexe Zusammenhänge aufscheinen lässt: Monokausale Erklärungen werden hinterfragt, und der Verlauf der ,Geschichte (wie ihn die traditionelle Historiographie rekonstruiert) wird zuweilen hypothetisch dezentriert. So löst der Legionär Titus Pullo (in der Serie die Buddy-Figur von Vorenus) einen folgenreichen Tumult aus: Als Marcus Antonius mit seinen Gefolgsleuten - darunter Pullo als sein Leibwächter - auf dem Weg zum Senat durch die Menge schreitet (Abb. 19), wird die Gruppe angegriffen, weil Pullo Tage zuvor in einer Taverne

33 Milo (1991) 18; 32; vgl. Späth / Tröhler (2012). 


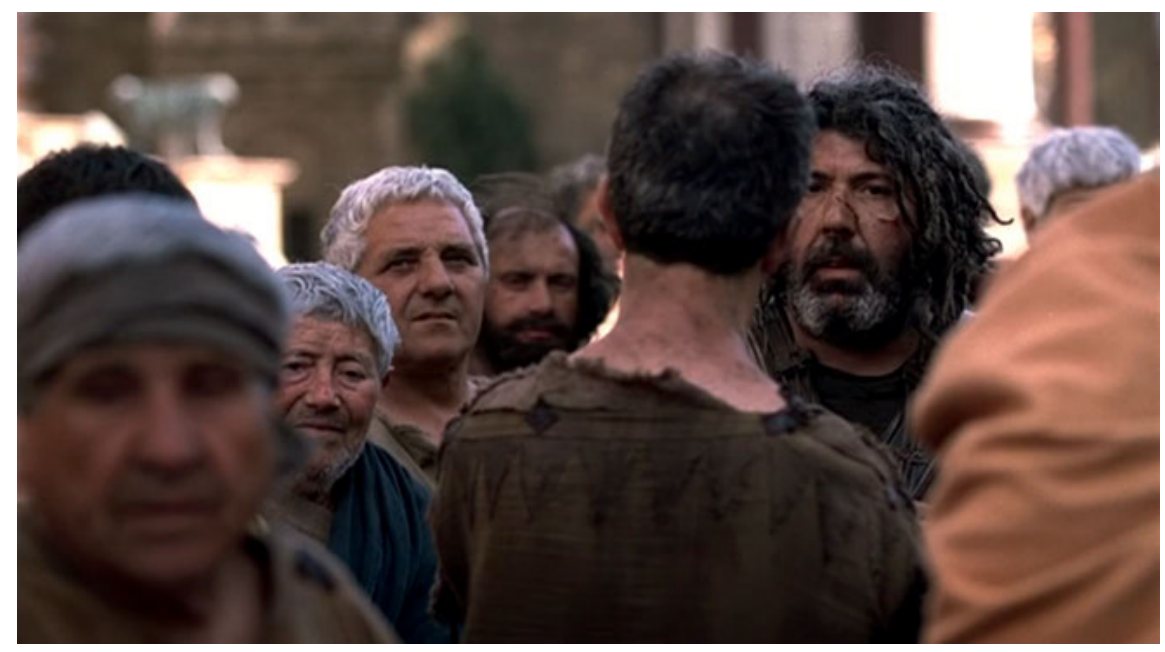

Abb. 20: Rome: Der Rächer in der Menge erblickt Pullo, ...

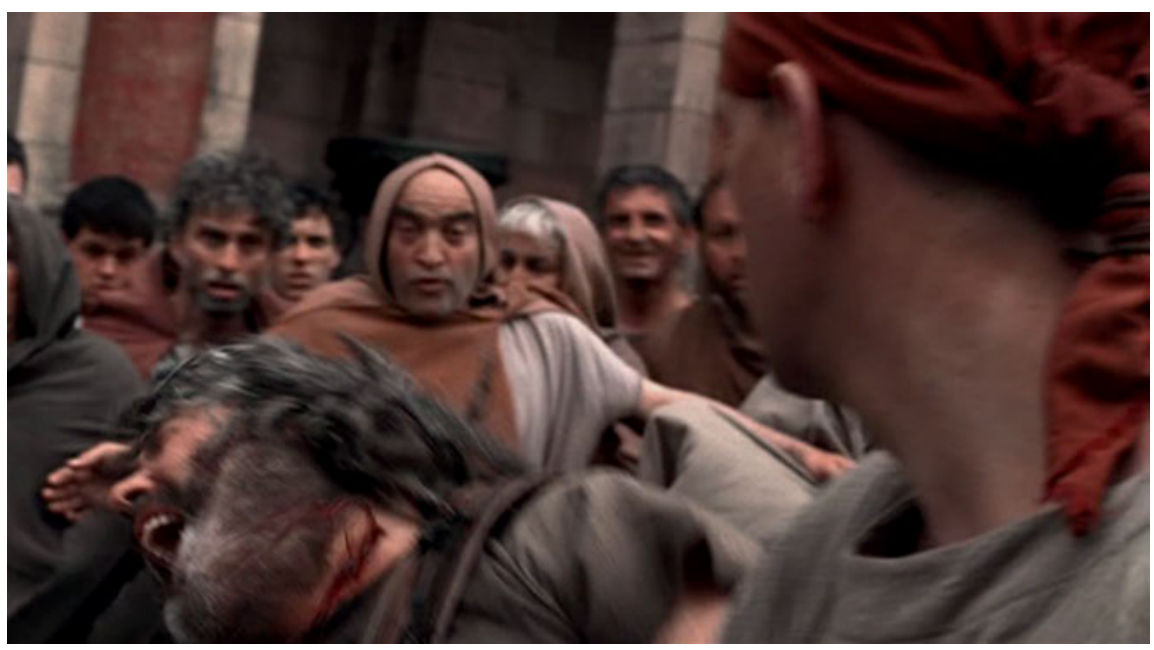

Abb. 21: Rome: ... greift ihn an und wird von ihm getötet.

auf dem Aventin einen Falschspieler erstochen hat, dessen Kumpane ihn nun rächen wollen (Abb. 20). Pompeius, Cato und Scipio, die sich unter den $\mathrm{Zu}$ schauern befinden, hatten zwar gegen Marcus Antonius intrigiert, doch ist nicht dieser, sondern Pullo der Grund der Anfeindung (Abb. 21-22). Er verantwortet damit letztlich den Sturz der Republik, weil Marcus Antonius den Senat nicht erreicht, wo er als Volkstribun sein Veto einlegen könnte, um zu verhin- 


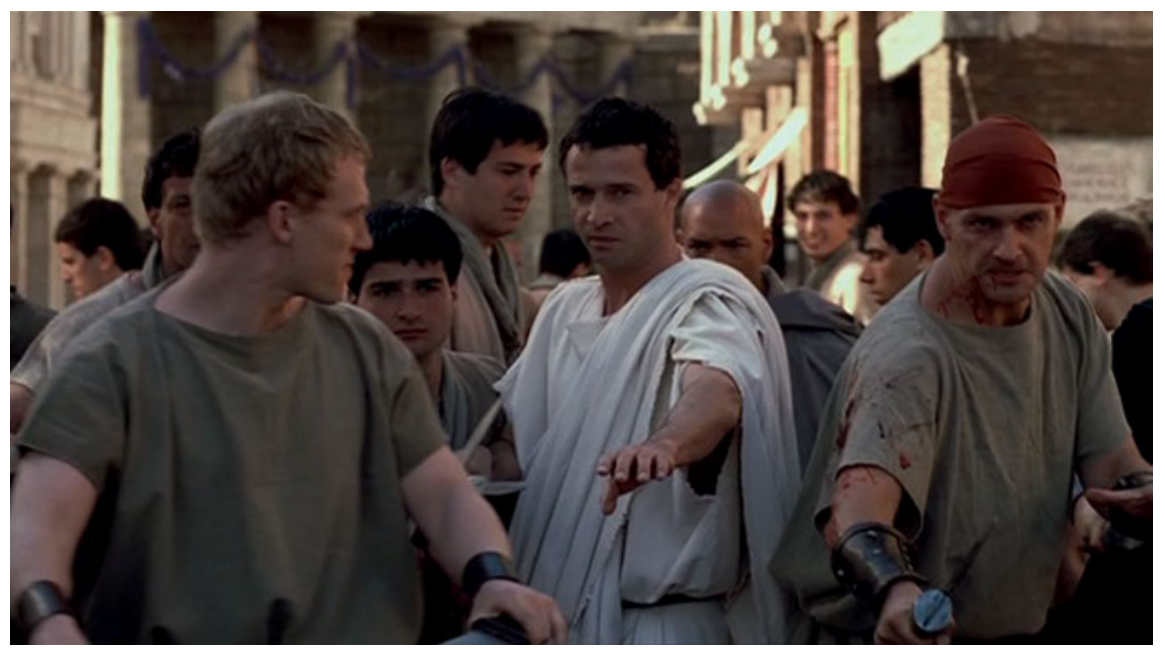

Abb. 22: Rome: Marcus Antonius zieht sich mit seinen Männern zurück - die Stadt bleibt durchwegs präsent.

dern, dass Caesar zum Staatsfeind erklärt wird. Die fiktive Figur eines einfachen Legionärs bewirkt somit, dass Caesar 49 v. Chr. den Rubikon überschreitet und nach Rom zurückkehrt.

Dies ist nur eine der unzähligen Situationen, die eine alternative Perspektive auf die historisch überlieferten Eckdaten und Heldentaten zulassen und zeigen, dass die Vorgänge, die zu historischen Ereignismomenten führen, nicht immer absichtsvoll geplant und von einem Einzelnen kontrolliert, sondern „kollektiv orchestriert“ sind. ${ }^{34}$ In dieser ironisch-selbstreflexiven Sichtweise auf die traditionelle Erzählung der Geschichte entwickelt die Serie eine Metaposition, die das kontingente Zusammenwirken verschiedener Kräfte und $\mathrm{Zu}$ fälle bei der Entstehung eines historischen Ereignisses erfahrbar macht. Die polyphone, horizontal-räumliche voranschreitende Erzählweise und die verkörperlichte diegetische Lebenswelt in Rome führen uns vor Augen, was Jameson, wie erwähnt, als ein ,Kartographieren der Wahrnehmung und Erkenntnis (im Sinne eines ,cognitive mapping') bezeichnet: Der urbane Raum als Ganzes, als Struktur, sowie die einzelnen Räume, Orte und Schauplätze der Stadt Rom erhalten durch die fiktiven alltäglichen Situationen eine ,physische Präsenz ${ }^{*}{ }^{35}$

34 Bourdieu (1980) 88.

35 Jameson (1993) 96 f. Auch Jameson dient die Matrix einer Stadt als Beispiel für eine räumliche Erzählweise, die das ,Kartographieren der Wahrnehmung und Erkenntnis` ermöglicht. Al- 
Mehr noch: Dieser Stadtraum generiert die (historischen) Konflikte und dient als Matrix für ein experimentelles historiographisches Modell der Antike, in dem Macht dezentral und Ereignisse in ihrer Kontingenz begründet sind. Durch die Fülle an audiovisuell wahrgenommenen Details und die komplexe Erzähldynamik tauchen wir als Zuschauer ein in die Entwicklung des Geschehens und sind gleichzeitig zum aktiven Kombinieren und Vergleichen aufgefordert, zum spielerischen, selbstreflexiven Formen und Umformen des imaginären Mosaiks des antiken Rom, des ikonographischen und narrativen Topos wie des gängigen Geschichtsbilds.

Die beiden hier vorgestellten Filmproduktionen erweitern das kulturell sedimentierte Gedächtnisbild der Stadt Rom und pars pro toto der Antike: Gladiator erneuert durch seine überwältigenden computergenerierten Bilder und die monumentale Rauminszenierung unsere Vorstellung von totalitärer Macht und deren Repräsentationsformen, während Rome durch die Konstruktion einer detailreichen und belebten Alltagswelt und die polyphone, verflachte Dynamik der Narration dem imaginären städtischen Raum in seiner strukturellen Komplexität eine physische Präsenz verschafft. In ihren Prozessen der (Re-)Semantisierung, der ästhetisch-narrativen Verräumlichung und der Diskursivierung der antiken Stadt Rom lassen sich indes sehr unterschiedliche historiographische Konzeptionen erkennen, die den Topos je auf ihre Weise in Schwingung versetzen.

\section{Literaturverzeichnis}

Barthes (1957): Roland Barthes, Mythologies, Paris.

Benjamin (1977a): Walter Benjamin, „Zentralpark“ (1938/39), in: Siegfried Unseld (Hg.),

Walter Benjamin. Illuminationen. Ausgewählte Schriften, Frankfurt a. M., 230-250.

Benjamin (1977b): Walter Benjamin, „Über den Begriff der Geschichte“ (1940), in: Siegfried

Unseld (Hg.), Walter Benjamin. Illuminationen. Ausgewählte Schriften, Frankfurt a. M., 251-261.

Blanchet u. a. (2011): Robert Blanchet, Kristina Köhler, Tereza Schmid u. Julia Zutavern

(Hgg.), Serielle Formen. Von den frühen Film-Serials zu aktuellen Quality-TV- und Online-

Serien, Marburg.

Bourdieu (1980): Pierre Bourdieu, Le sens pratique, Paris.

Eco (1985): Umberto Eco, „Innovation and Repetition: Between Modern and Post-Modern Aesthetics“, Daedalus 114/4, 161-184.

lerdings geht es ihm dabei nicht um ein historiographisches Modell, sondern um die „Neudefinition des Ideologiebegriffes“ (97), dessen Diskussion hier zu weit führen würde. 
Eigler (2005): Ulrich Eigler, „Barfuß durch die Sandalenfilme?“, in: Elisabeth Hermann-Otto

(Hg.), Unfreie Arbeits- und Lebensverhältnisse von der Antike bis zur Gegenwart,

Hildesheim / Zürich / New York, 357-370.

Genette (1982): Gérard Genette, Palimpseste. La littérature au second degré, Paris.

Jameson (1993): Fredric Jameson, „Postmoderne - zur Logik der Kultur im Spätkapitalismus“

(1984), in: Andreas Huyssen u. Klaus R. Scherpe (Hgg.), Postmoderne. Zeichen eines

kulturellen Wandels, Reinbek bei Hamburg, 45-102.

Lagny (1992): Michèle Lagny, „Popular Taste: The Peplum“, in: Richard Dyer u. Ginette

Vincendeau (Hgg.), Popular European Cinema, London / New York, 163-180.

Ludes (2011): Peter Ludes, Multimedia und Multi-Moderne: Schlüsselbilder. Fernseh-

nachrichten und World Wide Web-Medienzivilisierung in der europäischen

Währungsunion, Wiesbaden.

Milo (1991): Daniel S. Milo, „Pour une histoire expérimentale, ou le gai savoir“, in: Daniel

S. Milo u. Alain Boureau (Hgg.), Alter Histoire. Essais d'histoire expérimentale, Paris, 9-55.

Mittell (2006): Jason Mittell, „Narrative Complexity in Contemporary American Television“, The Velvet Light Trap. Review of Cinema 58/1, 29-40.

Pomeroy (2004): Arthur Pomeroy, „The Vision of a Fascist Rome in Gladiator“, in: Martin M. Winkler (Hg.), Gladiator. Film and History, Malden (Mass.) / Oxford, 111-123.

Rosen (2001): Philip Rosen, Change Mummified. Cinema Historicity, Theory, Minneapolis.

Rosenstone (1995): Robert A. Rosenstone, „History in Images / History in Words: Reflections on the Possibility of Really Putting History onto Film“ (1988), in: Robert Rosenstone, Visions of the Past. The Challenge of Film to Our Idea of History, Cambridge, 19-44.

Sabo (2012): Lee Weston Sabo: „Auteurs in the Arena. Antony Mann's Fall of the Roman Empire“, Bright Lights Film Journal, Mai, 76, o. S.; http://brightlightsfilm.com/76/ 76romanempire_sabo.php\#.UgJRHIPW9XY (30. Juli 2013).

Schöttker (2004): Detlev Schöttker, „Einführung. Benjamins Bilderwelten. Objekte, Theorien, Wirkungen“, in: Detlev Schöttker (Hg.), Schrift, Bilder, Denken. Walter Benjamin und die Künste, Berlin / Frankfurt a. M., 10-29.

Schweinitz (2006): Jörg Schweinitz, Film und Stereotyp. Eine Herausforderung für das Kino und die Filmtheorie. Zur Geschichte eines Mediendiskurses, Berlin.

Späth (2010): Thomas Späth, „In der Seele des Zenturio oder römische Geschichte als Soap“, in: Hans-Joachim Gehrke u. Miriam Sénécheau (Hgg.), Geschichte, Archäologie, Öffentlichkeit. Für einen neuen Dialog zwischen Wissenschaft und Medien. Standpunkte aus Forschung und Praxis, Bielefeld, 123-149.

Späth / Tröhler (2008): Thomas Späth u. Margrit Tröhler, „Spartacus - Männermuskeln, Heldenbilder oder: die Befreiung der Moral“, in: Tomas Lochman, Thomas Späth u. Adrian Stähli (Hgg.), Antike im Kino. Auf dem Weg zu einer Kulturgeschichte des Antikefilms, Basel, 170-193.

Späth / Tröhler (2012): Thomas Späth u. Margrit Tröhler, „Die TV-Serie Rome als Experimentelle Geschichtsschreibung “, Saeculum. Jahrbuch für Universalgeschichte 62,2, 267-302.

Stähli (2008): Adrian Stähli, „Die faschistische Antike im Film“, in: Tomas Lochman, Thomas Späth, Adrian Stähli (Hgg.), Antike im Kino. Auf dem Weg zu einer Kulturgeschichte des Antikefilms, Basel, 106-119.

Tatum (2008): Jeffrey W. Tatum, „Making History in Rome. Ancient vs. Modern Perspectives“, in: Monica Silveira Cyrino (Hg.), Rome, Season One. History Makes Television, Malden (Mass.), 29-41. 
Tröhler (2007): Margrit Tröhler, Offene Welten ohne Helden. Plurale Figurenkonstellationen im Film, Marburg.

Wieber (2012): Anja Wieber, „Film“, in: Heinz Heinen (Hg.; in Verbindung mit Ulrich Eigler et al.), Handwörterbuch der antiken Sklaverei (HAS), CD-ROM-Lieferung I-IV, Stuttgart.

Winkler (2009): Martin M. Winkler, „A Critial Appreciation of The Fall of the Roman Empire“, in: Martin M. Winkler (Hg.), The Fall of the Roman Empire: Film and History, Malden (Mass.) / Oxford, 1-50.

Winterling (2001): Aloys Winterling, „,Staat', ,Gesellschaft' und politische Integration in der römischen Kaiserzeit“, Klio 83, 93-112.

Winterling (2004): Aloys Winterling, „Zugänge zur Antike. Die antiken Menschen in ihren Gemeinschaften: Rom“, in: Eckard Wirbelauer (Hg.), Oldenbourg Geschichte Lehrbuch: Antike, München, 194-211.

Wulff (2002): Hans Jürgen Wulff, „Held und Antiheld, Prot- und Antagonist: Zur Texttheorie eines komplizierten Begriffsfeldes“, in: Hans Krah u. Claus-Michael Ort (Hgg.), Weltentwürfe in Literatur und Medien. Phantastische Wirklichkeiten - realistische Imaginationen, Festschrift für Marianne Wünsch, Kiel, 431-448. 
